Noname manuscript No.

(will be inserted by the editor)

\title{
Mathematical modeling of volumetric material growth
}

\author{
Jean-François Ganghoffer • Pavel I. \\ Plotnikov • Jan Sokołowski
}

Received: date / Accepted: date

\begin{abstract}
The model of volumetric material growth is introduced in the framework of finite elasticity. The new results obtained for the model are presented with complete proofs. The state variables include the deformations, temperature and the transplant matrix function. The wellposedness of the proposed model is shown. The existence of local in time classical solutions for the quasistatic deformations boundary value problem coupled with the energy balance and the growth evolution of the transplant is shown. The obtained mathematical results can be used for a wide class of growth models in mechanics and biology.
\end{abstract}

Keywords Volumetric growth · evolutionary problem · existence of local solutions

Mathematics Subject Classification (2000) 35Q74 - 35J60 - 35K61 . 34B15 - 35Q92

J.F. Ganghoffer

LEMTA, Nancy Université. 2, Avenue de la Forêt de Haye. BP 160. TSA 60604. 54518 Vandoeuvre Cedex. France

Tel.: +33-3-83595724

Fax: +33-3-83595551

E-mail: jean-francois.univ-lorraine.fr

P.I. Plotnikov

Lavrentyev Institute of Hydrodynamics, Lavrentyev pr. 15,630090 Novosibirsk, Russia

Tel.: +33-3-83595724

Fax: +33-3-83595551

E-mail: plotnikov@hydro.nsc.ru

J. Sokolowski

Institut Élie Cartan Nancy, UMR7502 (Université Lorraine, CNRS, INRIA), Laboratoire de Mathématiques, Université de Lorraine, B.P.239, 54506 Vandoeuvre-lès-Nancy Cedex, France

Tel.: $+33-3-83684580$

Fax: +33-3-83 684534

E-mail: Jan.Sokolowski@univ-lorraine.fr 


\section{Introduction}

\subsection{Motivation and main contribution}

The present work deals with the mathematical modeling of volumetric growth in thermoelastic bodies. The mechanical models are based on the general idea that growth can be taken into account by considering that deformations of a growing solid body are due to both changes of mass and elastic deformation. The most important statement of the theory from a kinematic viewpoint ([19]) is that the geometric deformation tensor is decomposed into the product of a growth tensor describing the local addition of material and an elastic tensor characterizing the reorganization of the body. The rigorous foundation of the volumetric growth theory was given in [7], with the so-called transplant tensor representing the growth transformation; our developments are based on the equations formulated in this last work. In this paper, we investigate the following questions:

Problem 1 In the framework of finite elasticity [27] we want to determine the state variables of growing elastic body including the deformation vector field $\mathbf{u}$, the scalar temperature $\theta$ and the so-called transplant mapping $K$ satisfying respectively the momentum balance equation, the energy balance equation, and the nonlinear evolutionary equation for the transplant field $\mathbf{K}$ supplemented with initial and boundary conditions.

Note 1 There are local, classical solutions to the model of growing, elastic body (3.5)-(3.6), when the initial transplant field has the form of the sum of a rotation and a bounded mapping. The precise mathematical result with the full proof is given by Theorem 1 .

\subsection{Short literature review}

Growth (resp. atrophy) describes the physical processes by which a material of solid body increases (resp. decreases) its size by addition (resp. removal) of mass. A clear distinction is generally made between growth per se, remodeling (change of properties), and morphogenesis (shape changes), a classification suggested by [24] Taber (1995). The advantages and drawbacks of the existing growth models are exposed in the recent contribution [14](Menzel and Kuhl, 2012). A first class of models is the kinematic models describing an evolution towards an homeostatic state rely on the kinematic decomposition of the transformation gradient into a generally incompatible mapping and an elastic mapping; they were historically introduced by [20] Rodriguez et al. (1994). The growth transformation evolves in time as a function of the difference between a stress measure and a corresponding measure associated to the surmised homeostatic state ([24] Taber, 1998 ; [21] Rodriguez et al., 2007; [1] Alford et al., 2008 ; [28] Vignes et Papadopoulos, 2010). This first class of models is criticized due to the absence of a rational mechanical framework. Approaches 
analogous to elastoplasticity have then been developed as a second class of models in a rational framework basing on the writing of the second principle of thermodynamics for open systems, in order to identify the evolution laws of growth ([12] Kuhl et al., 2007 ; [13] Menzel, 2007 ; [16] Olsson et Klarbring, 2008). It is important to note the prominent role of Eshelby stress in relation to the material driving forces for growth ([9], [10] Ganghoffer, 2010, 2011; [12] Kuhl et al., 2007), relying on Eshelby pioneering approach ([8] Eshelby, 1957). Central here is the idea to separate the shape variation due to the physical motion from the microstructural evolutions due to growth and remodeling phenomena occurring in the evolutive reference configuration.

\section{Mechanical background}

\subsection{Finite elasticity}

Constitution law. In this section we briefly discuss a basic facts from the finite elasticity theory. Throughout of the paper we shall assume that $\Omega \subset \mathbb{R}^{3}$ is a bounded reference domain with the boundary $\partial \Omega$ of class $C^{\infty}$ in the space of variable $x$. The state of an elastic material is characterized by a deformation field $\mathbf{u}=\left(u_{1}, u_{2}, u_{3}\right): \Omega \times[0, T] \rightarrow \mathbb{R}^{3}$ and the Kelvin temperature $\theta$ : $\Omega \times[0, T] \rightarrow \mathbb{R}^{+}$. The elastic distorsion tensor $D \mathbf{u}$ is the Jacobi matrix of the mapping $\mathbf{u}$ with the entries

$$
D \mathbf{u}_{i j}(x, t)=\partial_{j} u_{i}(x, t), \quad(x, t) \in \Omega \times[0, T]
$$

Here the notation

$$
\partial_{i}:=\partial_{x_{i}}=\partial / \partial x_{i}
$$

stands for the spatial derivatives. We will assume that the material is hyperelastic and it properties are described by the specific free energy density $\Psi(\theta, D \mathbf{u})$. In particular, a stress tensor $\mathbf{T}(\theta, D \mathbf{u})$ and internal energy $e(\theta, D \mathbf{u})$ are defined by the formulae

$$
\mathbf{T}(\theta, D \mathbf{u})=\frac{\partial \Psi(\theta, D \mathbf{u})}{\partial(D \mathbf{u})}, \quad e=\Psi(\theta, D \mathbf{u})-\theta \frac{\partial \Psi(\theta, D \mathbf{u})}{\partial \theta} .
$$

Here $\partial \Psi(\theta, \Phi) / \partial \Phi$ denotes the matrix with the entries

$$
\left(\frac{\partial \Psi(\theta, \Phi)}{\partial \Phi}\right)_{i j}=\frac{\partial \Psi(\theta, \Phi)}{\partial \Phi_{i j}}
$$

In many applications, it is sufficient to take the specific free energy density in the form

$$
\Psi(\theta, D \mathbf{u})=-c_{T} \theta \log \theta+\theta W(D \mathbf{u}),
$$

where $W$ is the stored elastic energy. The specific free energy density satisfies the two following conditions

$$
\begin{gathered}
\mathbf{T}(\theta, \Phi) \Phi^{\top}=\Phi \mathbf{T}(\theta, \Phi)^{\top} \\
\mathbf{T}(\theta, \mathbf{R} \Phi)=\mathbf{R T}(\theta, \Phi)
\end{gathered}
$$


for $\theta$, for all matrices $\Phi$, and for all orthogonal matrices R. Relation (2.3) expresses the angular momentum conservation law, and relation (2.4) expresses the observer independence principle.

Unstressed state and its stability. We assume that the reference configuration is unstressed, i.e.,

$$
\mathbf{T}(\theta, \mathbf{I})=0 \text { for all } \theta .
$$

It follows from (2.4) that

$$
\mathbf{T}(\theta, \mathbf{R})=0
$$

for all $\theta$ and all orthogonal matrices $\mathbf{R}$. In order to characterize stability properties of the reference configuration, it is convenient to introduce the linear matrix-valued form $L(\theta, \Phi)$ defined on the linear space of $3 \times 3$ matrices $\boldsymbol{\xi}$ by the equalities

$$
L(\theta, \Phi) \boldsymbol{\xi}=\frac{\partial \mathbf{T}(\theta, \Phi)}{\partial \Phi} \boldsymbol{\xi}=\frac{\partial \mathbf{T}(\theta, \Phi)}{\partial \Phi_{p q}} \xi_{p q} .
$$

Notice that $L(\theta, \Phi) \boldsymbol{\xi}$ is a matrix with the entries

$$
(L(\theta, \Phi) \boldsymbol{\xi})_{i j}=l_{i j p q}(\theta, \Phi) \xi_{p q}, \quad \text { where } l_{i j p q}(\theta, \Phi)=\frac{\partial^{2} \Psi(\theta, \Phi)}{\partial \Phi_{i j} \partial \Phi_{p q}} .
$$

The linear form $L$ is associated with the bilinear form

$$
L(\theta, \Phi) \boldsymbol{\xi} \cdot \boldsymbol{\eta}=l_{i j p q}(\theta, \Phi) \xi_{p q} \eta_{i j} .
$$

The following lemma will be used throughout the paper.

Lemma 1 For all $\theta$, for all matrices $\boldsymbol{\xi}, \boldsymbol{\eta}$, for all orthogonal matrices $\mathbf{R}$, and for all skew-symmetric matrices $\boldsymbol{\zeta}$,

$$
\begin{gathered}
L(\theta, \mathbf{R})(\boldsymbol{\xi} \mathbf{R}) \cdot(\boldsymbol{\eta} \mathbf{R})=L(\theta, \mathbf{I})\left(\mathbf{R}^{\top} \boldsymbol{\xi} \mathbf{R}\right) \cdot\left(\mathbf{R}^{\top} \boldsymbol{\eta} \mathbf{R}\right), \\
L(\theta, \mathbf{R})(\boldsymbol{\zeta} \mathbf{R})=0 \\
l_{i j p q}(\theta, \mathbf{R})=l_{\alpha j \sigma q}(\theta, \mathbf{I}) R_{p \sigma} R_{i \alpha}
\end{gathered}
$$

Proof It follows from (2.4) that

$$
\mathbf{T}(\theta, \mathbf{Z R})=\mathbf{R T}\left(\theta, \mathbf{R}^{-1} \mathbf{Z R}\right)=\mathbf{R} \mathbf{T}\left(\theta, \mathbf{R}^{\top} \mathbf{Z R}\right) .
$$

Choosing $\mathbf{Z}=\mathbf{I}+t \boldsymbol{\xi}$, we obtain

$$
\mathbf{T}(\theta, \mathbf{R}+t \boldsymbol{\xi} \mathbf{R})=\mathbf{R T}\left(\theta, \mathbf{I}+t \mathbf{R}^{\top} \boldsymbol{\xi} \mathbf{R}\right) .
$$

Differentiation both sides of this equality with respect to $t$ at $t=0$ gives

$$
\frac{\partial \mathbf{T}}{\partial \Phi}(\theta, \mathbf{R})(\boldsymbol{\xi} \mathbf{R})=\mathbf{R} \frac{\partial \mathbf{T}}{\partial \Phi}(\theta, \mathbf{I})\left(\mathbf{R}^{\top} \boldsymbol{\xi} \mathbf{R}\right) .
$$


Hence

$$
\begin{gathered}
L(\theta, \mathbf{R})(\boldsymbol{\xi R}) \cdot(\boldsymbol{\eta} \mathbf{R})=(\boldsymbol{\eta} \mathbf{R}) \cdot \frac{\partial \mathbf{T}}{\partial \Phi}(\theta, \mathbf{R})(\boldsymbol{\xi} \mathbf{R})=(\boldsymbol{\eta} \mathbf{R}) \cdot\left(\mathbf{R} \frac{\partial \mathbf{T}}{\partial \Phi}(\theta, \mathbf{I})\left(\mathbf{R}^{\top} \boldsymbol{\xi} \mathbf{R}\right)\right)= \\
\left(\mathbf{R}^{\top} \boldsymbol{\eta} \mathbf{R}\right) \cdot\left(\frac{\partial \mathbf{T}}{\partial \Phi}(\theta, \mathbf{I})\left(\mathbf{R}^{\top} \boldsymbol{\xi} \mathbf{R}\right)\right)=L(\theta, \mathbf{I})\left(\mathbf{R}^{\top} \boldsymbol{\xi} \mathbf{R}\right) \cdot\left(\mathbf{R}^{\top} \boldsymbol{\eta} \mathbf{R}\right),
\end{gathered}
$$

which gives (2.10). In order to prove (2.11), take an arbitrary skew-symmetric matrix $\boldsymbol{\zeta}$. Obviously, for every $t \in \mathbb{R}$, the matrices $\exp (t \boldsymbol{\zeta})$ and $\exp (t \boldsymbol{\zeta}) \mathbf{R}$ are orthogonal. From this and $(2.6)$, we obtain $\mathbf{T}(\theta, \exp (t \boldsymbol{\zeta}) \mathbf{R})=0$. Differentiating both sides of this equality with respect to $t$ at $t=0$ leads to (2.11). Next notice that relation (2.13) can be rewritten in the form

$$
l_{i j p \beta}(\theta, \mathbf{R}) R_{\mu \beta} \xi_{p \mu}=l_{\alpha j \sigma \beta}(\theta, \mathbf{I}) R_{i \alpha} R_{\mu \beta} R_{p \sigma} \xi_{p \mu},
$$

which gives

$$
\left(l_{i j p \beta}(\theta, \mathbf{R})-l_{\alpha j \sigma \beta}(\theta, \mathbf{I}) R_{i \alpha} R_{p \sigma}\right) R_{\mu \beta} \xi_{p \mu}=0
$$

Since $\boldsymbol{\xi}$ is an arbitrary matrix, we obtain

$$
\left(l_{i j p \beta}(\theta, \mathbf{R})-l_{\alpha j \sigma \beta}(\theta, \mathbf{I}) R_{i \alpha} R_{p \sigma}\right) R_{\mu \beta}=0 \text { for all } \mu, p
$$

Now fix an arbitrary $q$ and note that $R_{\mu \beta} R_{\mu q}=\delta_{\beta, q}$. It follows that

$$
l_{i j p q}(\theta, \mathbf{R})-l_{\alpha j \sigma q}(\theta, \mathbf{I}) R_{i \alpha} R_{p \sigma}=0,
$$

and the lemma follows.

We will assume throughout the paper that the specific energy satisfies the following stability condition

$$
L(\vartheta, \mathbf{I}) \boldsymbol{\xi} \cdot \boldsymbol{\xi} \geq c(\theta)\left|\boldsymbol{\xi}+\boldsymbol{\xi}^{\top}\right|^{2},
$$

for all matrices $\boldsymbol{\xi}$. Here the constant $c(\theta)$ is strongly positive and bounded for positive and bounded $\theta$.

Remark 1 It follows from stability condition (2.14) that for every orthogonal $\mathbf{R}$,

$$
L(\theta, \mathbf{R})(\boldsymbol{\xi} \mathbf{R}) \cdot(\boldsymbol{\xi} \mathbf{R}) \geq c(\theta)\left|\boldsymbol{\xi}+\boldsymbol{\xi}^{\top}\right|^{2} .
$$

Indeed, in view of Lemma 1 condition (2.14) and the identity

$$
\mathbf{R}^{\top} \boldsymbol{\xi} \mathbf{R}+\left(\mathbf{R}^{\top} \boldsymbol{\xi} \mathbf{R}\right)^{\top}=\mathbf{R}^{\top}\left(\boldsymbol{\xi}+\boldsymbol{\xi}^{\top}\right) \mathbf{R}
$$

we have

$$
\begin{array}{r}
L(\theta, \mathbf{R})(\boldsymbol{\xi R}) \cdot(\boldsymbol{\xi R})=L(\theta, \mathbf{I})\left(\mathbf{R}^{\top} \boldsymbol{\xi} \mathbf{R}\right) \cdot\left(\mathbf{R}^{\top} \boldsymbol{\xi} \mathbf{R}\right) \geq \\
c(\theta)\left|\mathbf{R}^{\top}\left(\boldsymbol{\xi}+\boldsymbol{\xi}^{\top}\right) \mathbf{R}\right|^{2}=c(\theta)\left|\boldsymbol{\xi}+\boldsymbol{\xi}^{\top}\right|^{2} .
\end{array}
$$

The following symmetry result is a well-known property of symmetry conditions (2.3)-(2.4) and equality (2.6), see [11], [27], [3] for the proofs.

Lemma 2 For every $\theta$,

$$
l(\theta, \mathbf{I})_{i j p q}=l(\theta, \mathbf{I})_{p q i j}=l(\theta, \mathbf{I})_{j i p q}=l(\theta, \mathbf{I})_{i j q p} .
$$




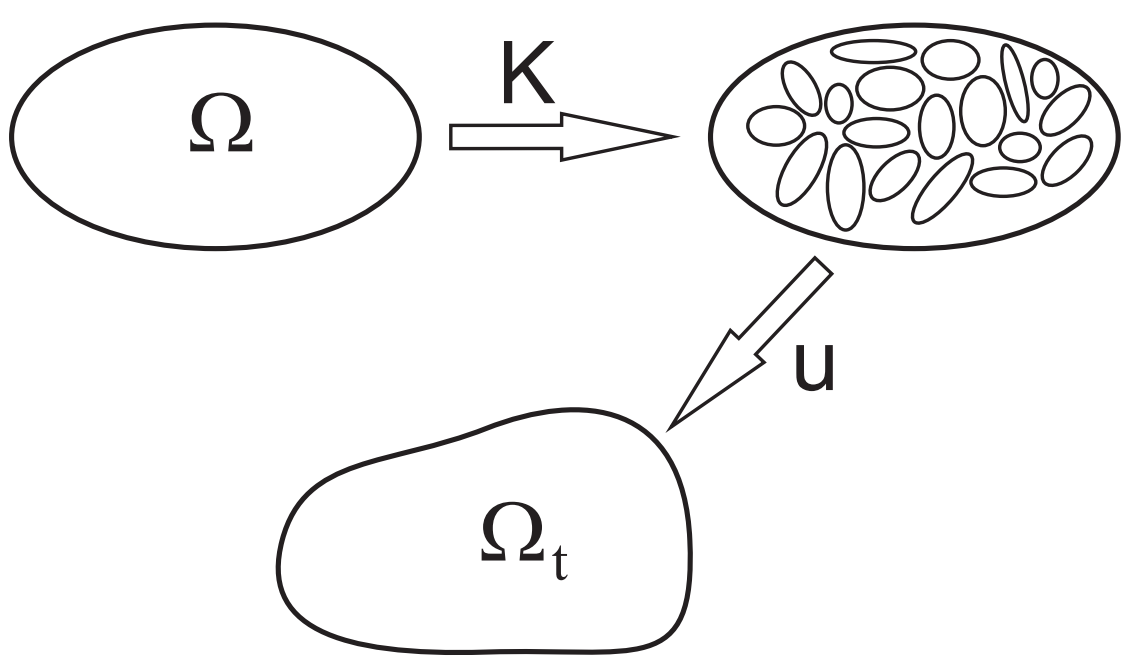

Fig. 1 Kinematics of volumetric growth: configurations and transplant mapping associated to growth.

\subsection{Growing material}

The main hypothesis of the volumetric growth theory is that a material consists of infinitesimally small particles $O(x, t)$ labeled by the reference coordinate $x$ and $t$. The growth of each particle is determined by the transplant matrix $\mathbf{K}(x, t): O(x, t) \rightarrow \mathbf{K}(x, t) O(x, t)$. Thus the distorsion tensor has the form of the product $D \mathbf{u K}$ of the elastic distorsion tensor $D \mathbf{u}$ and the transplant $\mathbf{K}$. The transplant tensor is responsible for material growth.

For growing materials, the specific free energy $\Psi_{g}(\theta, \mathbf{K}, D \mathbf{u})$, the stress tensor $\mathbf{T}_{g}(\theta, \mathbf{K}, D \mathbf{u})$, and the internal energy $e_{g}(\theta, \mathbf{K}, D \mathbf{u})$ are defined as follows.

$$
\begin{gathered}
\Psi_{g}(\theta, \mathbf{K}, D \mathbf{u})=\frac{1}{J_{K}} \Psi(\theta, D \mathbf{u K}), \\
\mathbf{T}_{g}(\theta, \mathbf{K}, D \mathbf{u})=\frac{1}{J_{K}} \frac{\partial \Psi(\theta, D \mathbf{u K})}{\partial(D \mathbf{u})}, \\
e_{g}(\theta, \mathbf{K}, D \mathbf{u})=\frac{1}{J_{K}}\left(\Psi(\theta, D \mathbf{u K})-\theta \frac{\partial \Phi(\theta, D \mathbf{u K})}{\partial \theta}\right) .
\end{gathered}
$$

Here $J_{K}=\operatorname{det} \mathbf{K}, \Psi$ is the specific free energy density of the basic elastic material. It is easy to see that

$$
\mathbf{T}_{g}(\theta, \mathbf{K}, D \mathbf{u})=\frac{1}{J_{K}} \frac{\partial \Psi(\theta, \Phi)}{\partial \Phi} \mathbf{K}^{\top}, \quad \text { where } \Phi=D \mathbf{u K} .
$$

If we take the specific free energy in the form

$$
\Psi_{g}(\theta, \mathbf{K}, D \mathbf{u})=\frac{1}{J_{K}}\left(-c_{T} \theta \log \theta+\theta W(D \mathbf{u K})\right),
$$


then we get the following expression for the stress tensor an the internal energy

$$
\mathbf{T}_{g}=\frac{\theta}{J_{K}} \frac{\partial W(\Phi)}{\partial \Phi} \mathbf{K}^{\top}, \quad \text { where } \Phi=D \mathbf{u} \mathbf{K}, \quad e_{g}=\frac{c_{T}}{J_{K}} \theta .
$$

The reference configuration is unstressed if and only if $\mathbf{K}=\mathbf{R}(x, t)$, where $\mathbf{R}$ is an orthogonal matrix. We stress that the tensor $\mathbf{K}$ is not potential, and $\mathbf{R}$ is an arbitrary orthogonal matrix depending on $(x, t)$. For given $\theta, \mathbf{K}$ and $D \mathbf{u}$, define the linear matrix-valued form $L_{g}(\theta, \mathbf{K}, D \mathbf{u})$ by the equality

$$
L_{g}(\theta, \mathbf{K}, D \mathbf{u}) \boldsymbol{\xi}=\lim _{\tau \rightarrow 0} \frac{1}{\tau}\left\{\mathbf{T}_{g}(\theta, \mathbf{K}, D \mathbf{u}+\tau \boldsymbol{\xi})-\mathbf{T}_{g}(\theta, \mathbf{K}, D \mathbf{u})\right\}
$$

Calculations shows that

$$
\left(L_{g}(\theta, \mathbf{K}, D \mathbf{u}) \boldsymbol{\xi}\right)_{i j}=l_{g, i j p q}(\theta, \mathbf{K}, D \mathbf{u}) \xi_{p q},
$$

where

$$
l_{g, i j p q}(\theta, \mathbf{K}, D \mathbf{u})=\frac{1}{J_{K}} \frac{\partial^{2} \Psi(\theta, \Phi)}{\partial \Phi_{i \alpha} \partial \Phi_{p \beta}} K_{j \alpha} K_{q \beta}, \quad \Phi=D \mathbf{u K} .
$$

The associated bilinear form is defined by

$$
L_{g}(\theta, \mathbf{K}, D \mathbf{u}) \boldsymbol{\xi} \cdot \boldsymbol{\eta}=l_{g, i j p q}(\theta, \mathbf{K}, D \mathbf{u}) \xi_{p q} \eta_{i j}
$$

It follows from $(2.22)$ that the forms $L_{g}$ and $L$ are connected by the relations

$$
L_{g}(\theta, \mathbf{K}, D \mathbf{u}) \boldsymbol{\xi} \cdot \boldsymbol{\eta}=L(\theta, \boldsymbol{\Phi})(\boldsymbol{\xi} \mathbf{K}) \cdot(\boldsymbol{\eta} \mathbf{K}), \quad \Phi=D \mathbf{u K}
$$

The following lemma is the extension of Lemma 1 to the case of growing materials.

Lemma 3 Let $\Psi$ satisfies symmetry conditions (2.3)-(2.4), equilibrium condition (2.6), and stability condition (2.14). Then, for all $\theta$, for all matrices $\boldsymbol{\xi}$, for all orthogonal matrices $\mathbf{R}$, and for all skew-symmetric matrices $\boldsymbol{\zeta}$,

$$
\begin{gathered}
L_{g}(\theta, \mathbf{R}, \mathbf{I}) \boldsymbol{\xi} \cdot \boldsymbol{\xi} \geq c(\theta)\left|\boldsymbol{\xi}+\boldsymbol{\xi}^{\top}\right|^{2}, \\
L_{g}(\theta, \mathbf{R}, \mathbf{I}) \boldsymbol{\zeta}=0, \\
l_{g, i j p q}(\theta, \mathbf{R}, \mathbf{I})=l_{\alpha m \sigma n}(\theta, \mathbf{I}) R_{i \alpha} R_{j m} R_{p \sigma} R_{q n} .
\end{gathered}
$$

Proof Notice that $J_{K}=1$ for $\mathbf{K}=\mathbf{R}$. It follows from this and (2.24) that

$$
L_{g}(\theta, \mathbf{R}, \mathbf{I}) \boldsymbol{\xi} \cdot \boldsymbol{\xi}=L(\theta, \mathbf{R})(\boldsymbol{\xi} \mathbf{R}) \cdot(\boldsymbol{\xi} \mathbf{R}) .
$$

From this and (2.10) we conclude that

$$
\begin{aligned}
& L_{g}(\theta, \mathbf{R}, \mathbf{I}) \boldsymbol{\xi} \cdot \boldsymbol{\xi}=L(\theta, I)\left(\mathbf{R}^{\top} \boldsymbol{\xi} \mathbf{R}\right) \cdot\left(\mathbf{R}^{\top} \boldsymbol{\xi} \mathbf{R}\right) \geq \\
& c(\theta)\left|\mathbf{R}^{\top} \boldsymbol{\xi} \mathbf{R}+\left(\mathbf{R}^{\top} \boldsymbol{\xi} \mathbf{R}\right) \top\right|^{2}=c(\theta)\left|\mathbf{R}^{\top}\left(\boldsymbol{\xi}+\boldsymbol{\xi}^{\top}\right) \mathbf{R}\right|^{2}=c(\theta)\left|\boldsymbol{\xi}+\boldsymbol{\xi}^{\top}\right|^{2}
\end{aligned}
$$


which leads to (2.25). Next, representation (2.21) implies

$$
\begin{aligned}
\left(L_{g}(\theta, \mathbf{K}, D \mathbf{u}) \boldsymbol{\xi}\right)_{i j}= & \frac{1}{J_{K}} \frac{\partial^{2} \Psi(\theta, \Phi)}{\partial \Phi_{i \alpha} \partial \Phi_{p \beta}} \xi_{p q} K_{q \beta} K_{j \alpha}= \\
& \frac{1}{J_{K}} l_{i \alpha p \beta}(\theta, \Phi)(\boldsymbol{\xi} \mathbf{K})_{p \beta} K_{j \alpha}=\frac{1}{J_{K}}(L(\theta, \Phi)(\boldsymbol{\xi} \mathbf{K}))_{i \alpha} K_{j \alpha},
\end{aligned}
$$

where $\Phi=D \mathbf{u K}$. Setting $\mathbf{K}=\mathbf{R}, D \mathbf{u}=\mathbf{I}$, and $\boldsymbol{\xi}=\boldsymbol{\zeta}$, we obtain

$$
\left(L_{g}(\theta, \mathbf{R}, \mathbf{I}) \boldsymbol{\zeta}\right)_{i j}=(L(\theta, \mathbf{R})(\boldsymbol{\zeta} \mathbf{R}))_{i \alpha} R_{j \alpha},
$$

which along with (2.11) yields (2.26). Next, it follows from representation (2.22) that

$$
l_{g, i j p q}(\theta, \mathbf{R}, \mathbf{I})=l_{i \alpha p \beta}(\theta, \mathbf{R}) R_{j \alpha} R_{q \beta} .
$$

Combining this result with (2.12), we obtain (2.27), which completes the proof.

Lemma 4 For every $\theta$,

$$
l_{g, i j p q}(\theta, \mathbf{R}, \mathbf{I})=l_{g, p q i j}(\theta, \mathbf{R}, \mathbf{I})=l_{g, j i p q}(\theta, \mathbf{R}, \mathbf{I})=l_{g, i j q p}(\theta, \mathbf{R}, \mathbf{I}) .
$$

Proof The proof obviously follows from (2.27) and the symmetry relations in Lemma 2.

\section{Problem formulation. Assumptions. Results}

Problem formulation. The problem consists of finding a deformation $\mathbf{u}$, a temperature $\theta$ and a transplant $\mathbf{K}$ satisfying the quasi-stationary momentum balance equation

$$
\operatorname{div} \mathbf{T}_{g}(\theta, \mathbf{K}, D \mathbf{u})+\mathbf{f}=0,
$$

the energy balance equation

$$
\frac{\partial}{\partial t} e_{g}(\theta, \mathbf{K}, D \mathbf{u})+\operatorname{div} \mathbf{q}=\mathbf{T}_{g}(\theta, \mathbf{K}, D \mathbf{u}) \cdot \frac{\partial}{\partial t}(D \mathbf{u}),
$$

and the evolutionary equation for $\mathbf{K}$,

$$
\frac{\partial}{\partial t} \mathbf{K}=\mathbf{g}(\theta, \mathbf{K}, \mathbf{u})
$$

Here $\mathbf{q}=\mathbf{q}(\nabla \theta, \theta, \mathbf{K}, D \mathbf{u})$ is a given heat flux, $\mathbf{g}$ a given matrix-valued function, $\mathbf{f}$ a given exterior dead force; the stress tensor $\mathbf{T}_{g}$ and the internal energy $e_{g}$ are defined by (2.16). Further, we assume that the free energy density is in the form (2.18) and take the heat flux in the simplest thermodynamically consistent form

$$
\mathbf{q}=\nabla\left(\frac{1}{\theta}\right)
$$

Thus, we obtain the following system of differential equations in the cylinder $\Omega \times(0, T)$

$$
\operatorname{div} \mathbf{T}_{g}(\theta, \mathbf{K}, D \mathbf{u})+\mathbf{f}=0 \text { in } \Omega \times(0, T),
$$




$$
\begin{gathered}
\frac{\partial}{\partial t}\left(\frac{c_{T} \theta}{J_{K}}\right)+\Delta\left(\frac{1}{\theta}\right)=\mathbf{T}_{g}(\theta, \mathbf{K}, D \mathbf{u}) \cdot \frac{\partial}{\partial t}(D \mathbf{u}) \text { in } \Omega \times(0, T) \\
\frac{\partial}{\partial t} \mathbf{K}=\mathbf{g}(\theta, \mathbf{K}, \mathbf{u}) \text { in } \Omega \times(0, T)
\end{gathered}
$$

Here

$$
\mathbf{T}_{g}=\frac{\theta}{J_{K}} \frac{\partial W(\Phi)}{\partial \Phi} \mathbf{K}^{\top}, \quad \Phi=D \mathbf{u} \mathbf{K} .
$$

These equations should be supplemented with boundary and initial conditions. For growing material, the problem of place with a fixed deformations of the boundary is not natural and we will instead consider the traction problem for the momentum equation

$$
-\mathbf{T}_{g}(\theta, \mathbf{K}, D \mathbf{u}) \mathbf{n}+\mathbf{h}=0 \quad \text { on } \partial \Omega \times(0, T)
$$

For simplicity we assume that there is no heat flux through the boundary, which leads to the following boundary condition for the temperature

$$
\nabla \theta \cdot \mathbf{n}=0 \text { on } \partial \Omega \times(0, T) .
$$

At the initial moment, the temperature and the transplant should be prescribed

$$
\theta(x, 0)=\Theta(x), \quad \mathbf{K}(x, 0)=\mathbf{K}_{0}(x) \text { in } \Omega .
$$

Here $\mathbf{n}$ is the unit outward normal vector to $\partial \Omega, \mathbf{h}, \Theta$ and $\mathbf{K}_{0}$ are given functions.

Before the formulation of results, it is convenient to introduce necessary notation and to formulate the assumptions on the boundary and initial data.

Definition 1 A couple $(\mathbf{f}, \mathbf{h}) \in C(\Omega) \times C(\partial \Omega)$ is said to be equilibrated if

$$
\begin{array}{r}
\int_{\Omega} \mathbf{f} d x+\int_{\partial \Omega} \mathbf{h} d s=0 \\
\int_{\Omega}\left(x_{i} f_{j}-x_{j} f_{i}\right) d x+\int_{\partial \Omega}\left(x_{i} h_{j}-x_{j} h_{i}\right) d s=0
\end{array}
$$

for all $i, j$.

Following [5], we introduce the astatic matrix $\mathbf{C}$ with the entries

$$
C_{i j}=\int_{\Omega} x_{i} f_{j} d x+\int_{\partial \Omega} x_{i} h_{j} d s .
$$

If the couple $(\mathbf{f}, \mathbf{h}) \in C(\Omega) \times C(\partial \Omega)$ is equilibrated, then the astatic matrix is symmetric.

Definition 2 An equilibrated couple $(\mathbf{f}, \mathbf{h}) \in C(\Omega) \times C(\partial \Omega)$ is said to be non-degenerate if there is a positive $c^{*}$ such that

$$
\left|\mu_{i}+\mu_{j}\right| \geq c^{*}\left(\|\mathbf{f}\|_{C(\Omega)}+\|\mathbf{h}\|_{C(\partial \Omega)}\right) \text { for all } i \neq j,
$$

where $\mu_{i}$ are eigenvalues of the matrix $\mathbf{C}$. 
Remark 2 An equilibrated couple $(\mathbf{f}, \mathbf{h}) \in C(\Omega) \times C(\partial \Omega)$ is non-degenerate if and only if for every skew-symmetric matrix $\mathbf{Y}$ the equations

$$
\mathbf{X C}-(\mathbf{X C})^{\top}=\mathbf{Y}, \quad \mathbf{X}=-\mathbf{X}^{\top}
$$

have a unique solution, which admits the estimate

$$
|\mathbf{X}| \leq\left(c^{*}\right)^{-1}\left(\|\mathbf{f}\|_{C(\Omega)}+\|\mathbf{h}\|_{C(\partial \Omega)}\right)^{-1}|\mathbf{Y}|
$$

Indeed, we can rewrite the first equation in the form $\mathbf{X C}+\mathbf{C X}=\mathbf{Y}$. Since $\mathbf{C}$ is symmetric, there is an orthogonal matrix $\mathbf{U}$ such that $\mathbf{U C \mathbf { U } ^ { \top }}=\mathbf{J}$, where $\mathbf{J}=\operatorname{diag}\left\{\mu_{i}\right\}$. Thus we get $\mathbf{X} \mathbf{U}^{\top} \mathbf{J U}+\mathbf{U}^{\top} \mathbf{J U X}=Y$, which leads to $\mathbf{Z J}+\mathbf{J Z}=\mathbf{H}$, with the skew symmetric matrices $\mathbf{Z}=\mathbf{U X U}^{\top}, \mathbf{H}=\mathbf{U Y U}^{\top}$. It remains to note that the latter equation can be written in the form

$$
\left(\mu_{1}+\mu_{2}\right) Z_{12}=H_{12}, \quad\left(\mu_{1}+\mu_{3}\right) Z_{13}=H_{13}, \quad\left(\mu_{2}+\mu_{3}\right) Z_{23}=H_{23} .
$$

Definition 3 For given constant $c^{*}>0$, denote by $\mathcal{F}_{c} \subset C(\Omega \times \partial \Omega)$ the set all equilibrated non-degenerate couples $(\mathbf{f}, \mathbf{h}) \neq 0$ satisfying inequality (3.9).

It is easily seen that zero is a limiting point of $\mathcal{F}_{c}$ and the set $\mathcal{F}$ is star-shaped, i.e., if $(\mathbf{f}, \mathbf{h}) \in \mathcal{F}_{c}$, then $\epsilon(\mathbf{f}, \mathbf{h}) \in \mathcal{F}_{c}$ for all $\epsilon \neq 0$. Finally, denote by $\mathcal{A}$ the annulus $\{1 / 2 \leq|| \Phi|-1| \leq 2\}$. We assume that the specific free energy, function $\mathrm{g}$ and the initial and boundary data satisfy the following conditions.

H.1 The specific free energy density $\Psi_{g}$ has the form

$$
\Psi_{g}(\theta, \mathbf{K}, D \mathbf{u})=\frac{1}{J_{K}}\left(-c_{T} \theta \log \theta+\theta W(D \mathbf{u K})\right) .
$$

The elastic stored energy $W \in C^{\infty}(\mathcal{A})$, and the matrix value function $\mathrm{g} \in C^{\infty}(\mathbb{R} \times \mathcal{A} \times \mathcal{A})$.

H.2 Let $\mathbf{T}$ be an elastic energy tensor with the entries $T_{i j}=\theta \partial W(\Phi) / \partial \Phi_{i j}$.

Then for all $\Phi \in \mathcal{A}$ and all orthogonal matrices $\mathbf{R}$,

$$
\mathbf{T}(\theta, \Phi) \Phi^{\top}=\Phi \mathbf{T}(\theta, \Phi)^{\top}, \quad \mathbf{T}(\theta, \mathbf{R} \Phi)=\mathbf{R} \mathbf{T}(\theta, \Phi), \quad \mathbf{T}(\theta, \mathbf{R})=0 .
$$

H.3 Let the bilinear form $L(\theta, \Phi)$ be defined by (2.8)-(2.9). Then

$$
L(\theta, \mathbf{I}) \boldsymbol{\xi} \cdot \boldsymbol{\xi} \geq c \theta\left|\boldsymbol{\xi}+\boldsymbol{\xi}^{\top}\right|^{2} \text { for all matrices } \boldsymbol{\xi} \text {. }
$$

H.4 There is $c^{*}>0$ such that $(\mathbf{f}(t), \mathbf{h}(t)) \in \mathcal{F}_{c}$ for every $t \in[0, T]$. The function $\Theta \in C^{\infty}(\Omega)$ satisfies the conditions

$$
0<c^{-1}<\Theta<c<\infty, \quad \partial_{n} \Theta=0 \text { on } \partial \Omega .
$$

The following theorem is the main result of this paper 
Theorem 1 Let $p>3$. Let conditions (H.1)-(H.4) be satisfied. Furthermore assume that

$$
\mathbf{K}_{0}(x)=\mathbf{R}(x)+\mathbf{k}_{0}(x),
$$

where $\mathbf{R} \in C^{\infty}(\Omega)$ is an arbitrary orthogonal matrix and $\mathbf{k}_{0} \in C^{\infty}(\Omega)$ is an arbitrary perturbation of $\mathbf{R}$. Then, there are $\lambda_{0}>0, \varepsilon_{0}>0$, and $T_{0}>0$ with the following property. For all $\lambda \in\left(0, \lambda_{0}\right]$, for all $\varepsilon \in\left(0, \varepsilon_{0}\right]$, for all $(\mathbf{f}, \mathbf{h}) \in \mathcal{F}_{c}$, and for all matrix-valued functions $\mathbf{k}_{0}$ satisfying

$$
\|\mathbf{f}\|_{C^{1}(0, T ; C(\Omega))}+\|\mathbf{h}\|_{C^{1}(0, T ; C(\partial \Omega))} \leq \varepsilon, \quad\left\|\mathbf{k}_{0}\right\|_{W^{p, 2}(\Omega)} \leq \lambda,
$$

problem (3.5)-(3.6) has a locally unique solution

$$
\begin{array}{r}
\mathbf{u} \in L^{\infty}\left(0, T_{0} ; W^{2, p}(\Omega)\right), \quad \mathbf{K} \in L^{\infty}\left(0, T_{0} ; W^{1, p}(\Omega)\right), \\
\theta \in L^{p}\left(0, T_{0} ; W^{2, p}(\Omega)\right)
\end{array}
$$

with

$$
\begin{aligned}
\partial_{t} \mathbf{u} \in L^{p}\left(0, T_{0} ; W^{1, p}(\Omega)\right), \partial_{t} \mathbf{K} & \in L^{p}\left(0, T_{0} ; W^{1, p}(\Omega)\right), \\
\partial_{t} \theta & \in L^{p}\left(0, T_{0} ; W^{1, p}(\Omega)\right) .
\end{aligned}
$$

The rest of the paper is devoted to the proof of this theorem. As will appear in the subsequent modified problem, the transplant field at any time (so not only tits initial value) shall be decomposed into a rotation and a deformation; this decomposition is in line with the fact that growth is defined up to a rotation, which can be accounted for in the accommodation mapping require to restore overall kinematic compatibility. We shall show that the decomposition holds only for bounded (small enough) transplant fields, so that it becomes additive.

Our strategy is the following. We are looking for a solution to problem (3.5)-(3.6) in the form $\mathbf{u}(x, t)=x+\mathbf{S}(t) x+\mathbf{v}(x, t)$, where $\mathbf{S}(t)$ is a skewsymmetric matrix and $\mathbf{v}$ is a vector field, which is orthogonal to rigid motions. Next, we exploit the method developed in [5] and replace problem (3.5)-(3.6) by the extended problem for $\mathbf{S}, \mathbf{v}$ and $\mathbf{K}, \theta$. Then we use the Implicit function Theorem in order to represent $\mathbf{S}$ and $\mathbf{v}$ as functions of $\theta$ and $\mathbf{K}$. Next, we substitute the result in the equations for $\theta$ and $\mathbf{K}$ in order to obtain the evolutionary system of operator equations for these functions. Finally, we solve the obtained evolutionary equations by using the method of successive approximations.

\section{Modified problem}

In this section, we formulate the extension of the basic equations (3.5)-(3.6). To this end, we introduce some auxiliary constructions. Following [5], we define 
the special nonlinear projection of the stress tensor on the space of equilibrated vector fields. Let choose an arbitrary vector field $\varphi \in C^{\infty}(\Omega)$ such that

$$
\begin{array}{r}
\int_{\Omega} \varphi d x=0, \quad \int_{\Omega} x_{i} \varphi_{j}(x)=0 \text { for all } i \neq j \\
\int_{\Omega}\left(x_{i} \varphi_{i}(x)+x_{j} \varphi_{j}(x)\right) d x \neq 0 \text { for all } i, j .
\end{array}
$$

Next we define the integral matrix-valued integral operator $\mathbf{E}(\theta, \mathbf{K}, D \mathbf{u})$ with the entries

$$
E_{i j}=\left(\int_{\Omega}\left(x_{i} \varphi_{i}+x_{j} \varphi_{j}\right) d x\right)^{-1} \int_{\Omega}\left(T_{g, i j}(\theta, \mathbf{K}, D \mathbf{u})-T_{g, j i}(\theta, \mathbf{K}, D \mathbf{u})\right) d x .
$$

Finally set

$$
\mathcal{E}(\theta, \mathbf{K}, D \mathbf{u})=\mathbf{E}(\theta, \mathbf{K}, D \mathbf{u}) \boldsymbol{\varphi} .
$$

Recall that the temperature $\theta$ coincides with the given smooth strictly positive function $\Theta(x)$ at the initial moment. We set

$$
\theta(x, t)=\Theta(x)+\vartheta(x, t),
$$

where $\vartheta$ is a new unknown function. Finally, for every positive $T_{0}$, we set

$$
\chi(t)=1 \text { for } t \leq T_{0}, \quad \chi(t)=0 \text { for } t>T_{0} .
$$

We are now in a position to formulate the modified system of equations and boundary and initial conditions. Since $\theta(x, 0)=\Theta(x)$ and $\mathbf{K}(x, 0)=\mathbf{R}(x)+$ $\mathbf{k}_{0}(x)$ it is convenient to formulate the problem in terms of the perturbations

$$
\vartheta(x, t):=\theta(x, t)-\Theta(x), \quad \mathbf{k}(x, t)=\mathbf{K}(x, t)-\mathbf{R}(x)
$$

The functions $\vartheta$ and $\mathbf{k}$ are considered as new unknowns and should be defined along with a solution to the problem We are now in a position to formulate the modified problem.

Problem M. For given $T \geq T_{0}>0,(\mathbf{f}, \mathbf{h}), \Theta(x), \mathbf{R}(x)$, and $\mathbf{k}_{0}(x)$, find a deformation field $\mathbf{u}$, a temperature $\theta$, and a transplant $\mathbf{K}$ which admit the representation

$$
\begin{aligned}
\mathbf{u}(x, t)=x+\mathbf{S}(t) x+\mathbf{v}(x, t) & \text { in } \Omega \times(0, T), \\
\theta(x, t)=\Theta(x)+\vartheta(x, t), \quad \mathbf{K}(x, t)=\mathbf{R}(x)+\mathbf{k}(x, t) & \text { in } \Omega \times(0, T),
\end{aligned}
$$

where $\mathbf{S}(t)$ is an unknown skew-symmetric matrix and $\mathbf{v}$ satisfies the conditions

$$
\int_{\Omega} \mathbf{v}(x, t) d x=0, \quad \int_{\Omega}\left(\partial_{i} v_{j}(x, t)-\partial_{j} v_{i}(x, t)\right) d x=0
$$

for all $i j$ and $t \in[0, T]$. The functions $\vartheta, \mathbf{k}, \mathbf{v}$, and the matrix $\mathbf{S}$ should satisfy the static equations

$$
\operatorname{div} T_{g}(\theta, \mathbf{K}, D \mathbf{u})+\mathcal{E}(\theta, \mathbf{K}, D \mathbf{u})+f=0 \text { in } \Omega \times(0, T),
$$




$$
\begin{gathered}
-T_{g}(\theta, \mathbf{K}, D \mathbf{u}) \mathbf{n}+\mathbf{h}=0 \text { on } \partial \Omega \times(0, T), \\
\mathbf{S C}-(\mathbf{S C})^{\top}+\mathbf{H}(\mathbf{v})-\mathbf{H}^{\top}(\mathbf{v})=0 \text { in }(0, T),
\end{gathered}
$$

and the evolutionary equations

$$
\begin{array}{r}
c_{T} \frac{\partial \vartheta}{\partial t}-\Delta\left(\frac{\vartheta}{\Theta^{2}}\right)=\chi(t)\left\{c_{T} \frac{\partial \vartheta}{\partial t}-\Delta\left(\frac{\vartheta}{\Theta^{2}}\right)-c_{T} \frac{\partial}{\partial t}\left(\frac{\theta}{J_{K}}\right)-\right. \\
\left.\Delta\left(\frac{1}{\theta}\right)+\mathbf{T}(\vartheta, \mathbf{K}, D \mathbf{u}) \cdot D \frac{\partial \mathbf{u}}{\partial t}\right\} \text { in } \Omega \times(0, T),
\end{array}
$$

$$
\nabla \vartheta \cdot \mathbf{n}=0 \text { on } \partial \Omega \times(0, T), \quad \vartheta(x, 0)=0 \text { in } \Omega
$$

$$
\frac{\partial \mathbf{k}}{\partial t}=\chi(t) \mathbf{g}(\theta, \mathbf{R}+\mathbf{k}, D \mathbf{u}) \quad \text { in } \quad \Omega \times(0, T), \quad \mathbf{k}(x, 0)=\mathbf{k}_{0}(x) \text { in } \Omega .
$$

Here $\mathbf{C}(t)$ and $\mathbf{H}$ are matrices with the entries

$$
C_{i j}=\int_{\Omega} x_{i} f_{j} d x+\int_{\partial \Omega} x_{i} h_{j} d s, \quad H_{i j}=\int_{\Omega} v_{i} f_{j} d x+\int_{\partial \Omega} v_{i} h_{j} d s .
$$

Our next task is to prove the local solvability of problem (4.5) and to show that this solution satisfies equations (3.5)-(3.6). We start with the analysis of the static problem.

\section{Static problem}

In this section, we consider the following stationary traction problem for a skew symmetric matrix $\mathbf{S}$ and the vector field $\mathbf{v}(x)$.

$$
\begin{gathered}
\operatorname{div} T_{g}(\theta, \mathbf{K}, D \mathbf{u})+\mathcal{E}(\theta, \mathbf{K}, D \mathbf{u})+f=0 \text { in } \Omega \times(0, T), \\
-T_{g}(\theta, \mathbf{K}, D \mathbf{u}) \mathbf{n}+\mathbf{h}=0 \text { on } \partial \Omega \times(0, T), \\
\mathbf{S C}-(\mathbf{S C})^{\top}+\mathbf{H}(\mathbf{v})-\mathbf{H}^{\top}(\mathbf{v})=0 \text { in }(0, T), \\
\mathbf{u}(x)=x+\mathbf{S} x+\mathbf{v}(x) \text { in } \Omega \times(0, T), \\
\int_{\Omega} \mathbf{v}(x, t) d x=0, \quad \int_{\Omega}\left(\partial_{i} v_{j}(x, t)-\partial_{j} v_{i}(x, t)\right) d x=0
\end{gathered}
$$

Here the load $(\mathbf{f}, \mathbf{h})$, the transplant $\mathbf{K}$, and the temperature $\theta$ are considered as given functions of variable $x$. We employ the Implicit function theorem in order to show that problem (5.1) has a strong solution which is locally unique and continuously depends on $\theta$ and $\mathbf{K}$. Before doing so, we define the special Banach spaces of functions of the variable $x \in \Omega$. 
Definition 4 For every $p \in(1, \infty)$, let denote by $\mathbb{X}_{p}$ the closed subspace of $W^{2, p}(\Omega)$ which consists of all functions $\mathbf{v}: \Omega \rightarrow \mathbb{R}^{3}$ satisfying the conditions

$$
\int_{\Omega} \mathbf{v} d x=0, \quad \int_{\Omega}\left(\partial_{i} v_{j}-\partial_{j} v_{i}\right) d x=0 \text { for all } i j .
$$

Definition 5 For every $p \in(1, \infty)$, we denote by $\mathbb{Y}_{p}$ the Banach space which consists of all couples $(\mathbf{f}, \mathbf{h})$ which are equilibrated and have the finite norm

$$
\|\mathbf{f}, \mathbf{h}\|_{\mathbb{Y}_{p}}=\|\mathbf{f}\|_{L^{p}(\Omega)}+\|\mathbf{h}\|_{W^{1-1 / p, p}(\Omega)} .
$$

Definition 6 Denote by $\mathbb{S}_{3}$ the Banach space of all skew-symmetric matrices. The space $\mathbb{S}_{3}$ is topologically and algebraically isomorphic to $\mathbb{R}^{3}$.

Fix $p>3$ and denote by $B(r), W(r), V(r)$, and $\Sigma(r)$ the balls

$$
\begin{array}{r}
B(r)=\left\{\theta:\|\theta\|_{W^{1, p}(\Omega)} \leq r\right\}, \\
V(r)=\left\{\mathbf{v} \in \mathbb{X}_{p}:\|\mathbf{v}\|_{\mathbb{X}_{p}} \leq r\right\}, \\
\Sigma(r)=\left\{\mathbf{S} \in \mathbb{S}_{3}:|\mathbf{S}| \leq r\right\} .
\end{array}
$$

The following theorem on solvability of problem (5.1) is the main result of this section

Theorem 2 Let $p>3$. Let conditions (H.1)-(H.4) be satisfied. Furthermore, assume that

$$
\mathbf{K}(x)=\mathbf{R}(x)+\mathbf{k}(x), \quad \theta(x)=\Theta(x)+\vartheta(x) .
$$

Then there are positive $\epsilon_{0}, \lambda_{0}$ and $\mu>0$ with the following properties. For every $\lambda \in\left(0, \lambda_{0}\right]$ and $\varepsilon \in\left(0, \varepsilon_{0}\right]$ there is $\mu(\varepsilon, \lambda)>0$ such that for all $\mathbf{k} \in B(\lambda)$, $\vartheta \in B(\lambda)$ and for every $(\mathbf{f}, \mathbf{h}) \in \mathcal{F}_{c}$ with

$$
0<\|\mathbf{f}\|_{C(\Omega)}+\|\mathbf{h}\|_{C(\partial \Omega)} \leq \varepsilon,
$$

problem (5.1) has a unique solution

$$
\mathbf{v}=\mathcal{V}(\vartheta, \mathbf{k}), \quad \mathbf{S}=\mathcal{S}(\vartheta, \mathbf{k})
$$

in the ball $V(\mu) \times \Sigma(\mu)$. The operators $\mathcal{V}: B(\lambda) \times B(\lambda) \rightarrow V(\mu), \mathcal{S}: B(\lambda) \times$ $B(\lambda) \rightarrow \Sigma(\mu)$ are infinitely differentiable and $\mu=\mu(\varepsilon, \lambda) \rightarrow 0$ as $(\varepsilon, \lambda) \rightarrow$ $(0,0)$. This solution admits the estimates

$$
\begin{gathered}
\|\mathcal{V}(0,0)\|_{\mathbb{X}_{p}}+|\mathcal{S}(0,0)| \leq c \varepsilon, \\
\left\|\mathcal{V}\left(\vartheta^{\prime}, \mathbf{k}^{\prime}\right)-\mathcal{V}\left(\vartheta^{\prime \prime}, \mathbf{k}^{\prime \prime}\right)\right\|_{\mathbb{x}_{p}}+\left|\mathcal{S}\left(\vartheta^{\prime}, \mathbf{k}^{\prime}\right)-\mathcal{S}\left(\vartheta^{\prime \prime}, \mathbf{k}^{\prime \prime}\right)\right| \leq \\
c\left(\left\|\vartheta^{\prime}-\vartheta^{\prime \prime}\right\|_{W^{1, p}(\Omega)}+\left\|\mathbf{k}^{\prime}-\mathbf{k}^{\prime \prime}\right\|_{W^{1, p}(\Omega)}\right),
\end{gathered}
$$

for all $\vartheta^{\prime}, \vartheta^{\prime \prime} \in B(\lambda)$ and $\mathbf{k}^{\prime}, \mathbf{k}^{\prime \prime} \in B(\lambda)$. Here the constant $c$ is independent of $\varepsilon$ and $\lambda$. Moreover, the identity

$$
\mathcal{E}(\Theta+\vartheta, \mathbf{R}+\mathbf{k}, \mathbf{I}+\mathcal{S}(\vartheta, \mathbf{k})+D \mathcal{V}(\vartheta, \mathbf{k}))=0
$$

holds for all $(\vartheta, \mathbf{k}) \in B(\lambda) \times B(\lambda)$. 
As a corollary, we obtain the following result on the solvability of the quasistatic problem.

Theorem 3 Let $p>3$. Let conditions (H.1)-(H.4) be satisfied. Furthermore assume that

$$
\begin{aligned}
\mathbf{K}(x, t)=\mathbf{R}(x)+\mathbf{k}(x, t), & \theta(x, t)=\Theta(x)+\vartheta(x, t), \\
\mathbf{k}(\cdot, t) \in B(\lambda), & \vartheta(\cdot, t) \in B(\lambda), \\
\partial_{t} \vartheta \in L^{p}\left(0, T ; W^{1, p}(\Omega)\right), & \partial_{t} \mathbf{k} \in L^{p}\left(0, T ; W^{1, p}(\Omega)\right),
\end{aligned}
$$

where $B(\lambda)$ is defined in Theorem 2. We also assume that for every $t \in(0, T)$ the couple $(\mathbf{f}, \mathbf{h}) \in C^{1}(0, T ; C(\Omega) \times C(\partial \Omega))$ satisfies the conditions

$$
\begin{gathered}
(\mathbf{f}(t), \mathbf{h}(t)) \in \mathcal{F}_{c}, \quad\|\mathbf{f}(t)\|_{C(\Omega)}+\|\mathbf{h}(t)\|_{C(\partial \Omega)} \leq \varepsilon \leq \varepsilon_{0}, \\
\left\|\partial_{t} \mathbf{f}(t)\right\|_{C(\Omega)}+\left\|\partial_{t} \mathbf{h}(t)\right\|_{C(\partial \Omega)} \leq c\left(\|\mathbf{f}(t)\|_{C(\Omega)}+\|\mathbf{h}(t)\|_{C(\partial \Omega)}\right),
\end{gathered}
$$

where $\varepsilon_{0}$ is defined by Theorem 2. Then, for every $t \in(0, T)$ problem (5.1) has a unique solution $(\mathbf{v}(t), \mathbf{S}(t)) \in V(\mu) \times \Sigma(\mu)$. This solution has the representation

$$
\mathbf{v}=\mathcal{V}(\vartheta, \mathbf{k}, t), \quad \mathbf{S}=\mathcal{S}(\vartheta, \mathbf{k}, t)
$$

in which the operators $\mathcal{V}(\cdot, t): B(\lambda) \times B(\lambda) \rightarrow V(\mu), \mathcal{S}(\cdot, t): B(\lambda) \times B(\lambda) \rightarrow$ $\Sigma(\mu)$ are infinitely differentiable, admit the estimates (5.6), and satisfy identity (5.8). Moreover the function

$$
\mathbf{u}(x, t)=x+\mathcal{S}(\vartheta, \mathbf{k}, t) x+\mathcal{V}(\vartheta, \mathbf{k}, t)
$$

has the time derivative

$$
\partial_{t} \mathbf{u}=\mathcal{P}(\vartheta, \mathbf{k}, t)+\mathcal{Q}_{1}(\vartheta, \mathbf{k}, t) \partial_{t} \vartheta+\mathcal{Q}_{2}(\vartheta, \mathbf{k}, t) \partial_{t} \mathbf{k}
$$

The operators $\mathcal{P}, \mathcal{Q}_{i}$ satisfy the inequalities

$$
\begin{gathered}
\left\|\mathcal{P}\left(\vartheta_{1}, \mathbf{k}_{1}, t\right)-\mathcal{P}\left(\vartheta_{2}, \mathbf{k}_{2}, t\right)\right\|_{W^{2, p}(\Omega)} \leq \\
c\left(\left\|\vartheta_{1}-\vartheta_{2}\right\|_{W^{1, p}(\Omega)}+\left\|\mathbf{k}_{1}-\mathbf{k}_{2}\right\|_{W^{1, p}(\Omega)}\right), \\
\left\|\mathcal{Q}_{i}\left(\vartheta_{1}, \mathbf{k}_{1}, t\right) f-\mathcal{Q}_{i}\left(\vartheta_{2}, \mathbf{k}_{2}, t\right) f\right\|_{W^{2, p}(\Omega)} \leq \\
c\left(\left\|\vartheta_{1}-\vartheta_{2}\right\|_{W^{1, p}(\Omega)}+\left\|\mathbf{k}_{1}-\mathbf{k}_{2}\right\|_{W^{1, p}(\Omega)}\right)\|f\|_{W^{1, p}(\Omega)}, \\
\left\|\mathcal{P}\left(\vartheta_{1}, \mathbf{k}_{1}, t\right)\right\|_{W^{2, p}(\Omega)} \leq c(\varepsilon+\lambda),
\end{gathered}
$$

for all $t \in(0, T), \vartheta_{j} \in B(\lambda), \mathbf{k}_{j} \in B(\lambda)$, and $f \in L^{p}(\Omega)$.

The rest of the section is devoted to the proof of Theorems 2 and 3 . 


\subsection{Proof of Theorem 2}

We reduce the static boundary value problem (5.1) to an operator equation.

To this end, we introduce the operators

$$
\begin{gathered}
\Xi_{0}(\mathbf{v}, \mathbf{S}, \vartheta, \mathbf{k})=\operatorname{div} \mathbf{T}_{g}(\Theta+\vartheta, \mathbf{R}+\mathbf{k}, \mathbf{I}+\mathbf{S}+D \mathbf{v})+ \\
\mathcal{E}(\Theta+\vartheta, \mathbf{R}+\mathbf{k}, \mathbf{I}+\mathbf{S}+D \mathbf{v})+\mathbf{f}, \\
\Xi_{b}(\mathbf{v}, \mathbf{S}, \vartheta, \mathbf{k})=-\mathbf{T}_{g}(\Theta+\vartheta, \mathbf{R}+\mathbf{k}, \mathbf{I}+\mathbf{S}+D \mathbf{v}) \mathbf{n}+\left.\mathbf{h}\right|_{\partial \Omega}, \\
\Xi_{e}(\mathbf{v}, \mathbf{S})=\frac{1}{\|\mathbf{f}\|_{C(\Omega)}+\|\mathbf{h}\|_{C(\partial \Omega)}}\left\{\mathbf{S C}-(\mathbf{S C})^{\top}+\mathbf{H}(\mathbf{v})-\mathbf{H}(\mathbf{v})^{\top}\right\}, \\
\Xi=\left(\Xi_{0}, \Xi_{b}, \Xi_{e}\right) .
\end{gathered}
$$

Thus we rewrite (5.1) in the form of the operator equation

$$
\Xi(\mathbf{v}, \mathbf{S}, \vartheta, \mathbf{k})=0 .
$$

We apply the Implicit function Theorem in order to resolve this equation with respect to $\mathbf{v}$ and $\mathbf{S}$. To this end, we have to check that the operator $\Xi$ is smooth and meets all requirements of the Implicit function Theorem. We split the proof of these facts into a sequence of lemmas. Recall Definitions 4-6 for spaces $\mathbb{X}_{p}, \mathbb{Y}_{p}, \mathbb{S}_{3}$ and definition (5.3) of balls $B(r) \subset W^{1, p}(\Omega), V(r) \subset \mathbb{Y}_{p}$, and $\Sigma_{r} \subset \mathbb{S}_{3}$.

Lemma 5 Under the assumptions of Theorem 2, there is $r>0$ such that the operator

$$
\Xi: V(r) \times \Sigma(r) \times B(r) \times B(r) \rightarrow \mathbb{Y}_{p} \times \mathbb{S}_{3}
$$

is infinitely differentiable.

Proof Since $p>3$, the embedding $\mathbb{Y}_{p} \hookrightarrow C^{1}(\Omega), W^{1, p}(\Omega) \hookrightarrow C(\Omega)$ is continuous. Hence we can choose $r$ so small that

$$
\left|(\mathbf{R}+\mathbf{k})^{ \pm 1}\right| \leq 2, \quad 1 / 2 \leq|(\mathbf{I}+\mathbf{S}+D \mathbf{v})(\mathbf{R}+\mathbf{k})| \leq 2
$$

for all $\mathbf{k} \in B(r), \mathbf{v} \in V(r)$, and $\mathbf{S} \in \Sigma(r)$. Next notice that

$$
\mathbf{T}_{g}(\theta, \mathbf{K}, D \mathbf{u})=\frac{\Theta+\vartheta}{\operatorname{det}(\mathbf{R}+\mathbf{k})} \frac{\partial W}{\partial \Phi}((\mathbf{I}+\mathbf{S}+D \mathbf{v})(\mathbf{R}+\mathbf{k}))(\mathbf{R}+\mathbf{k})^{\top} .
$$

The right hand side can be regarded as a function of the entries of matrices $\mathbf{R}, \mathbf{k}, \mathbf{S}, D \mathbf{v}$. It follows from Condition (H.1) that this function is infinitely differentiable on the range of matrices $\mathbf{k} \in B(r), \mathbf{v} \in V(r)$, and $\mathbf{S} \in \Sigma(r)$. The classical results of the finite elasticity theory, see [3], [27] impliy that the operator

$$
V(r) \times \Sigma(r) \times B(r) \times B(r) \ni(\mathbf{v}, \mathbf{S}, \vartheta, \mathbf{k}) \mapsto \mathbf{T}_{g}(\theta, \mathbf{K}, D \mathbf{u}) \in W^{1, p}(\Omega),
$$


is infinitely differentiable. Since the embedding $W^{1, p}(\Omega) \hookrightarrow W^{1-1 / p, p}(\partial \Omega)$ is continuous, the operator

$$
\left(\Xi_{0}, \Xi_{b}\right): V(r) \times \Sigma(r) \times B(r) \times B(r) \rightarrow L^{p}(\Omega) \times W^{1-1 / p, p}(\partial \Omega)
$$

is infinitely differentiable. The operator $\Xi_{e}: V(r) \times \Sigma(r) \rightarrow \mathbb{S}_{3}$ is linear and continuous. Hence it is obviously infinitely differentiable. It remains to prove that the operator $\left(\Xi_{0}, \Xi_{b}\right)$ takes its values in the space of $\mathbb{Y}_{p}$, which is a subspace of $L^{p}(\Omega) \times W^{1-1 / p, p}(\partial \Omega)$, see Definition 5 . This fact is a straightforward consequence of the choice of the projection $\mathcal{E}$. It is suffices to prove that the couple $\left(\Xi_{0}, \Xi_{b}\right)$ is equilibrated. We begin with the observation that in view of (4.1)-(4.3),

$$
\int_{\Omega} \Xi_{0} d x+\int_{\partial \Omega} \Xi_{b} d s=\int_{\Omega} \operatorname{div} \mathbf{T}_{g} d x-\int_{\partial \Omega} \mathbf{T}_{g} \mathbf{n} d s+E \int_{\Omega} \boldsymbol{\varphi} d x \equiv 0
$$

Next we have

$$
\begin{array}{r}
\int_{\Omega}\left(x_{j} \Xi_{0, i}-x_{i} \Xi_{0}, j\right) d x+\int_{\partial \Omega}\left(x_{j} \Xi_{b, i}-x_{i} \Xi_{b j}\right) d s= \\
\int_{\Omega}\left(x_{j} \partial_{p}\left(T_{g, i p}\right)-x_{i} \partial_{q}\left(T_{g, j q}\right)\right) d x-\int_{\partial \Omega}\left(x_{j} T_{g, i p} n_{p}-x_{i} T_{g, j q} n_{q}\right) d s+ \\
\int_{\Omega}\left(E_{i p} x_{j} \varphi_{p}-E_{j q} x_{i} \varphi_{q}\right) d x .
\end{array}
$$

Integrating by part the right hand side, we obtain

$$
\begin{aligned}
& \int_{\Omega}\left(x_{j} \Xi_{0, i}-x_{i} \Xi_{0}, j\right) d x+\int_{\partial \Omega}\left(x_{j} \Xi_{b, i}-x_{i} \Xi_{b j}\right) d s= \\
& -\int_{\Omega}\left(T_{g, i j}-T_{g, j i}\right) d x+\int_{\Omega}\left(E_{i p} x_{j} \varphi_{p}-E_{j q} x_{i} \varphi_{q}\right) d x
\end{aligned}
$$

Since the matrix $\mathbf{E}$ is independent of $x$, it follows from (4.1) and (4.2) that

$\int_{\Omega}\left(E_{i p} x_{j} \varphi_{p}-E_{j q} x_{i} \varphi_{q}\right) d x=\int_{\Omega}\left(E_{i j} x_{j} \varphi_{j}-E_{j i} x_{i} \varphi_{i}\right) d x=\int_{\Omega}\left(T_{g, i j}-T_{g, j i}\right) d x$.

Substituting this equality into (5.21) we obtain

$$
\int_{\Omega}\left(x_{j} \Xi_{0, i}-x_{i} \Xi_{0}, j\right) d x+\int_{\partial \Omega}\left(x_{j} \Xi_{b, i}-x_{i} \Xi_{b j}\right) d s=0 .
$$

Combining this result with $(5.20)$, we conclude that the couple $\left(\Xi_{0}, \Xi_{b}\right)$ is equilibrated; this completes the proof.

In order to apply the Implicit function theorem we have to prove that the partial derivative $D_{(\mathbf{v}, \mathbf{S})} \Xi$ is invertible at the zero point; hence our next task 
is to calculate this derivative. Recall that the derivative $D_{(\mathbf{v}, \mathbf{S})} \Xi(\mathbf{v}, \mathbf{S}, \vartheta, \mathbf{k})$ is a linear operator which acts on couples $(\mathbf{w}, \boldsymbol{\xi}) \in \mathbb{X}_{p} \times \mathbb{S}_{3}$ by the formula

$$
D_{(\mathbf{v}, \mathbf{S})} \Xi(\mathbf{v}, \mathbf{S}, \vartheta, \mathbf{k})[\mathbf{w}, \boldsymbol{\xi}]=\lim _{\tau \rightarrow 0} \frac{1}{\tau}\{\Xi(\mathbf{v}+\tau \mathbf{w}, \mathbf{S}+\tau \boldsymbol{\xi}, \vartheta, \mathbf{k})-\Xi(\mathbf{v}, \mathbf{S}, \vartheta, \mathbf{k})\}
$$

Now recall definition (2.20) of the linear form $L_{g}$. It follows from (2.21) that

$$
\left(L_{g}(\Theta, \mathbf{R}, \mathbf{I}) D \mathbf{w}\right)_{i j}=l_{g, i j p q}(\Theta, \mathbf{R}, \mathbf{I}) \partial_{q} w_{p},
$$

where the coefficients $l_{g, i j p q}$ are given by (2.22).

Lemma 6 Under the assumptions of Theorem 2,

$$
\begin{aligned}
& D_{(\mathbf{v}, \mathbf{S})} \Xi(0,0,0,0)[\mathbf{w}, \boldsymbol{\xi}]= \\
& \quad\left(\operatorname{div}\left(L_{g}(\Theta, \mathbf{R}, \mathbf{I}) D \mathbf{w}\right),-\left.L_{g}(\Theta, \mathbf{R}, \mathbf{I}) D \mathbf{w n}\right|_{\partial \Omega}, \Xi_{e}(\mathbf{w}, \boldsymbol{\xi})\right) .
\end{aligned}
$$

Proof We begin with calculation of the derivatives of operators $\mathbf{T}_{g}$ and $\mathcal{E}$. We have

$$
\begin{gathered}
D_{(\mathbf{v}, \mathbf{S})} \mathbf{T}_{g}(\Theta, \mathbf{R}, \mathbf{I})[\mathbf{w}, \boldsymbol{\xi}]=\lim _{\tau \rightarrow 0} \frac{1}{\tau}\left\{\mathbf{T}_{g}(\Theta, \mathbf{R}, \mathbf{I}+\tau \boldsymbol{\xi}+\tau D \mathbf{w})-\mathbf{T}_{g}(\Theta, \mathbf{R}, \mathbf{I})\right\} \equiv \\
L_{g}(\Theta, \mathbf{R}, \mathbf{I})(\boldsymbol{\xi}+D \mathbf{w})=L_{g}(\Theta, \mathbf{R}, \mathbf{I}) D \mathbf{w}
\end{gathered}
$$

since $L_{g}(\Theta, \mathbf{R}, \mathbf{I}) \boldsymbol{\xi}=0$ for any skew-symmetric matrix $\boldsymbol{\xi}$. It follows from this and expression (4.2) for the entries of the matrix $\mathbf{E}$ that

$$
\begin{gathered}
D_{(\mathbf{v}, \mathbf{S})} E_{i j}(\Theta, \mathbf{R}, \mathbf{I})[\mathbf{w}, \boldsymbol{\xi}]=\lim _{\tau \rightarrow 0} \frac{1}{\tau}\left\{E_{i j}(\Theta, \mathbf{R}, \mathbf{I}+\tau \boldsymbol{\xi}+\tau D \mathbf{w})-E_{i j}(\Theta, \mathbf{R}, \mathbf{I})\right\}= \\
\left\{\int_{\Omega}\left(x_{i} \varphi_{i}+x_{j} \varphi_{j}\right) d x\right\}^{-1} \int_{\Omega}\left(\left(L_{g}(\Theta, \mathbf{R}, \mathbf{I}) D \mathbf{w}\right)_{i j}-\left(L_{g}(\Theta, \mathbf{R}, \mathbf{I}) D \mathbf{w}\right)_{j i}\right) d x .
\end{gathered}
$$

Next notice that in view of (5.23) and Lemma 4,

$$
\begin{aligned}
& \left(L_{g}(\Theta, \mathbf{R}, \mathbf{I}) D \mathbf{w}\right)_{i j}-\left(L_{g}(\Theta, \mathbf{R}, \mathbf{I}) D \mathbf{w}\right)_{j i}= \\
= & \left(l_{g, i j p q}(\Theta, \mathbf{R}, \mathbf{I})-l_{g, j i p q}(\Theta, \mathbf{R}, \mathbf{I})\right) \partial_{q} w_{p}=0 .
\end{aligned}
$$

Hence $D_{(\mathbf{v}, \mathbf{S})} E_{i j}(\Theta, \mathbf{R}, \mathbf{I})=0$. It follows from this that $D_{(\mathbf{v}, \mathbf{S})} \mathcal{E}(\Theta, \mathbf{R}, \mathbf{I})=0$. Combining this result with (5.25) and (5.18a)-(5.18b), we obtain

$$
\begin{aligned}
D_{(\mathbf{v}, \mathbf{S})}\left(\Xi_{0}(0,0,0,0),\right. & \Xi_{b}(0,0,0,0)[\mathbf{w}, \boldsymbol{\xi}]= \\
& \left(\operatorname{div}\left(L_{g}(\Theta, \mathbf{R}, \mathbf{I}) D \mathbf{w}\right),-\left.L_{g}(\Theta, \mathbf{R}, \mathbf{I}) D \mathbf{w n}\right|_{\partial \Omega}\right) .
\end{aligned}
$$

It remains to note that the operator $\Xi_{e}$ is linear and coincides with its derivative. This completes the proof. 
Lemma 7 . Let $\Theta \in C^{\infty}(\Omega), 0<c^{-1} \leq \Theta \leq c$, and $\mathbf{R} \in C^{\infty}(\Omega)$ be a field of orthogonal matrices. Then there is $c>0$ such that for every $(\mathbf{F}, \mathbf{H}) \in \mathbb{Y}_{p}$ the boundary value problem

$$
\begin{aligned}
& \operatorname{div}\left(L_{g}(\Theta, \mathbf{R}, \mathbf{I}) D \mathbf{w}\right)+\mathbf{F}=0 \quad \text { in } \Omega, \\
& -L_{g}(\Theta, \mathbf{R}, \mathbf{I}) D \mathbf{w n}+\mathbf{H}=0 \quad \text { on } \partial \Omega
\end{aligned}
$$

has a unique solution $\mathbf{w} \in \mathbb{X}_{p}$. This solution admits the estimate

$$
\|\mathbf{w}\|_{\mathbb{X}_{p}} \leq c\|(\mathbf{F}, \mathbf{H})\|_{\mathbb{Y}_{p}} .
$$

Proof It follows from (5.23) that equations (5.28) form a second order system of partial differential equations with the Neumann type boundary condition

$$
\begin{gathered}
\partial_{j}\left(l_{g, i j p q}(\Theta, \mathbf{R}, \mathbf{I}) \partial_{q} w_{p}\right)+F_{i}=0 \text { in } \Omega, \\
-\left(l_{g, i j p q}(\Theta, \mathbf{R}, \mathbf{I}) \partial_{q} w_{p}\right) n_{j}+H_{i}=0 \text { on } \partial \Omega
\end{gathered}
$$

In view of the conditions of the lemma, the coefficients of these equations are smooth in $\Omega$. The problems of this type were thoroughly investigated in [26] and we simply recall the corresponding result. Along with problem (5.29) we will consider the homogeneous boundary problem

$$
\begin{gathered}
\partial_{j}\left(l_{g, i j p q}(\Theta, \mathbf{R}, \mathbf{I}) \partial_{q} w_{p}\right)=0 \text { in } \Omega, \\
-\left(l_{g, i j p q}(\Theta, \mathbf{R}, \mathbf{I}) \partial_{q} \cdot w_{p}\right) n_{j}=0 \text { on } \partial \Omega
\end{gathered}
$$

Let us show that problem (5.27) define the nonnegative quadratic form. To this end notice that in view of $(2.25)$ the inequality

$$
\begin{array}{r}
\left.\int_{\Omega} \operatorname{div}\left(L_{g}(\Theta, \mathbf{R}, \mathbf{I}) D \boldsymbol{\psi}\right) \cdot \boldsymbol{\psi} d x-\int_{\Omega}\left(L_{g}(\Theta, \mathbf{R}, \mathbf{I}) D \boldsymbol{\psi}\right]\right) \mathbf{n} \cdot \boldsymbol{\psi} d s= \\
\int_{\Omega} L_{g}(\Theta, \mathbf{R}, \mathbf{I}) D \boldsymbol{\psi} \cdot D \boldsymbol{\psi} d x \geq c(\Theta) \int_{\Omega}\left|D \boldsymbol{\psi}+(D \boldsymbol{\psi})^{\top}\right|^{2} d x
\end{array}
$$

holds for all smooth functions $\boldsymbol{\psi}$. From this and Korn inequality, we obtain that for every smooth $\psi$,

$$
\begin{array}{r}
\int_{\Omega} \operatorname{div}\left(L_{g}(\Theta, \mathbf{R}, \mathbf{I}) D \boldsymbol{\psi}\right) \cdot \boldsymbol{\psi} d x-\int_{\Omega}\left(L_{g}(\Theta, \mathbf{R}, \mathbf{I}) D \boldsymbol{\psi}\right) \mathbf{n} \cdot \boldsymbol{\psi} d s+ \\
\int_{\Omega}|\boldsymbol{\psi}|^{2} d x \geq c\|\boldsymbol{\psi}\|_{W^{1,2}(\Omega)}^{2}
\end{array}
$$

From this and Theorem 12, [26], we conclude that boundary problem (5.29) is elliptic and the boundary condition satisfies the completing conditions. The general theory of elliptic boundary value problems implies that in this case any weak solution $\mathbf{w} \in W^{1,2}(\Omega)$ to problem (5.29) satisfies the estimate

$$
\|\mathbf{w}\|_{W^{2, p}(\Omega)} \leq c\left(\|\mathbf{F}\|_{L^{p}(\Omega)}+\|\mathbf{H}\|_{W^{1-1 / p, p}(\partial \Omega)}+\|\mathbf{w}\|_{W^{1,2}(\Omega)}\right),
$$


where $c$ is independent of $\mathbf{w}, \mathbf{F}$, and $\mathbf{H}$. Moreover, see [26], problem (5.29) has a weak solution $\mathbf{w} \in W^{1,2}(\Omega)$ for every couple $(\mathbf{F}, \mathbf{H}) \in W^{1,2}(\Omega)^{\prime} \times W^{-1 / 2,2}(\Omega)$ satisfying the solvability condition

$$
\left\langle\mathbf{F}, \mathbf{w}^{*}\right\rangle+\left\langle\mathbf{H}, \mathbf{w}^{*}\right\rangle=0,
$$

for all solutions $\mathbf{w}^{*} \in W^{1,2}(\Omega)$ of the transposed homogeneous problem. Since the transposed problem to (5.29) is elliptic and satisfies the completing condition, we have $\mathbf{w}^{*} \in C^{\infty}(\Omega)$. On the other hand, problem (5.29) is symmetric and hence $\mathbf{w}^{*}$ satisfies equations and boundary conditions (5.30). From this and estimate (5.31), we conclude that $D \mathbf{w}^{*}+\left(D \mathbf{w}^{*}\right)^{\top}=0$. Hence $\mathbf{w}^{*}=$ const. $+\mathbf{S}^{*} x$, where $\mathbf{S}^{*}$ is an arbitrary skew symmetric matrix. On the other hand, in view of Lemma 3 , we have $L_{g}(\Theta, \mathbf{R}, \mathbf{I}) \mathbf{S}^{*}=0$. Hence all solutions to the homogeneous transposed problem are in the form $\mathbf{w}^{*}=$ const. $+\mathbf{S}^{*} x$. In this case, the solvability condition (5.33) simply means that the couple $(\mathbf{F}, \mathbf{H})$ is equilibrated. From this and Definition 5 of the Banach space $\mathbb{Y}_{p}$, we conclude that for every $(\mathbf{F}, \mathbf{H}) \in \mathbb{Y}_{p}$, problem (5.29) has a solution $\mathbf{w} \in W^{2, p}(\Omega)$ satisfying (5.32). This solution is not unique. However, we can choose $\mathbf{w}^{*}=$ const. $+\mathbf{S}^{*} x$ in such a way that after change of variable $\mathbf{w} \rightarrow \mathbf{w}-\mathbf{w}^{*}$, the function $\mathbf{w}$ will satisfy the conditions

$$
\int_{\Omega} \mathbf{w} d x=0, \quad \int_{\Omega} D \mathbf{w} d x \text { is symmetric, }
$$

i.e. $\mathbf{w} \in \mathbb{X}_{p}$. Because of Korn inequality, such a solution is unique and hence

$$
\|\mathbf{w}\|_{\mathbb{X}_{p}}=\|\mathbf{w}\|_{W^{2, p}(\Omega)} \leq c\|(\mathbf{F}, \mathbf{H})\|_{\mathbb{Y}_{p}} .
$$

This completes the proof.

Lemma 8 Under the assumptions of Theorem 2, the operator $D_{(\mathbf{v}, \mathbf{S})} \Xi(0,0,0,0)$ has a continuous inverse

$$
D_{(\mathbf{v}, \mathbf{S})} \Xi(0,0,0,0)^{-1}: \mathbb{Y}_{p} \times \mathbb{S}_{3} \rightarrow \mathbb{X}_{p} \times \mathbb{S}_{3},
$$

which norm is bounded by the constant independent of $\mathbf{f}$ and $\mathbf{h}$.

Proof Choose an arbitrary $(\mathbf{F}, \mathbf{H}) \in \mathbb{Y}_{p}$ and $\mathbf{G} \in \mathbb{S}_{3}$. It suffices to prove that the equation

$$
D_{(\mathbf{v}, \mathbf{S})} \Xi(0,0,0,0)[\mathbf{w}, \boldsymbol{\zeta}]=(\mathbf{F}, \mathbf{H}, \mathbf{G})
$$

has a unique solution $(\mathbf{w}, \boldsymbol{\zeta}) \in \mathbb{X}_{p} \times \mathbb{S}_{3}$, and this solution admits the estimate

$$
\|\mathbf{w}\|_{\mathbb{X}_{p}}+|\boldsymbol{\zeta}| \leq c\left(\|(\mathbf{F}, \mathbf{H})\|_{\mathbb{Y}_{p}}+|\mathbf{G}|\right) .
$$

In view of Lemma equation (5.34) and formula (5.18c), equation (5.34) can be written in the form

$$
\begin{aligned}
& \operatorname{div}\left(L_{g}(\Theta, \mathbf{R}, \mathbf{I}) D \mathbf{w}\right)-\mathbf{F}=0 \text { in } \Omega, \\
& -L_{g}(\Theta, \mathbf{R}, \mathbf{I}) D \mathbf{w n}-\mathbf{H}=0 \text { on } \partial \Omega
\end{aligned}
$$




$$
\zeta \mathbf{C}-(\boldsymbol{\zeta C})^{\top}=-\mathbf{H}(\boldsymbol{w})+\mathbf{H}(\boldsymbol{w})^{\top}+\left(\|\mathbf{f}\|_{C(\Omega)}+\|\mathbf{h}\|_{C(\partial \Omega)}\right) \mathbf{G}
$$

It follows from Lemma 7 that the boundary value problem (5.36) has a unique solution $\mathbf{w} \in \mathbb{X}_{p}$ such that

$$
\|\mathbf{w}\|_{\mathbb{X}_{p}} \leq c\|(\mathbf{F}, \mathbf{H})\|_{\mathbb{Y}_{p}} .
$$

Next, it follows from Remark 2 that the matrix equation (5.37) has a unique solution which satisfies the inequalities

$$
|\boldsymbol{\zeta}| \leq c|\mathbf{G}|+c\left(\|\mathbf{f}\|_{C(\Omega)}+\|\mathbf{h}\|_{C(\partial \Omega)}\right)^{-1}|\mathbf{H}(\mathbf{w})| .
$$

On the other hand, expression (4.5i) for $\mathbf{H}$ gives

$$
\begin{aligned}
|\mathbf{H}(\mathbf{w})| & \leq \int_{\Omega}|\mathbf{f}||\mathbf{w}| d x+\int_{\partial \Omega}|\mathbf{h} \| \mathbf{w}| d s \leq \\
c\left(\|\mathbf{f}\|_{C(\Omega)}+\|\mathbf{h}\|_{C(\partial \Omega)}\right)\|\mathbf{w}\|_{C(\Omega))} & \leq c\left(\|\mathbf{f}\|_{C(\Omega)}+\|\mathbf{h}\|_{C(\partial \Omega)}\right)\|\mathbf{w}\|_{\mathbb{X}_{p}} .
\end{aligned}
$$

Thus we get

$$
|\boldsymbol{\zeta}| \leq c|\mathbf{G}|+c\|\mathbf{w}\|_{\mathbb{X}_{p}}
$$

Combining this estimate with (5.38) we arrive at (5.35), and the lemma follows.

Proof of Theorem 2 We are now in a position to complete the proof of Theorem 2. By Lemma 5, the operator

$$
\Xi: V(r) \times \Sigma(r) \times B(r) \times B(r) \rightarrow \mathbb{Y}_{p} \times \mathbb{S}_{3} .
$$

is infinitely differentiable, and the norms of its derivatives depend only on $\Theta, \mathbf{R}, \Omega, p$ and $r$. Next, by Lemma 8 , the operator $D_{(\mathbf{v}, \mathbf{S})} \Xi(0,0,0,0)$ has a bounded inverse

$$
D_{(\mathbf{v}, \mathbf{S})} \Xi(0,0,0,0)^{-1}: \mathbb{Y}_{p} \times \mathbb{S}_{3} \rightarrow \mathbb{X}_{p} \times \mathbb{S}_{3}
$$

Finally,

$$
\|\Xi(0,0,0,0)\|_{\mathbb{Y}_{p} \times \mathbb{S}_{3}}=\|(\mathbf{f}, \mathbf{h}, 0)\|_{\mathbb{Y}_{p} \times \mathbb{S}_{3}} \leq \varepsilon .
$$

Hence, $\Xi$ satisfies all conditions of the Implicit function Theorem. Applying this theorem, we conclude that there are positive $\epsilon_{0}, \lambda_{0}$ with the following properties. For every $\lambda \in\left(0, \lambda_{0}\right]$ and $\varepsilon \in\left(0, \varepsilon_{0}\right]$ there is $\mu(\varepsilon, \lambda)>0$ such that for all $\mathbf{k} \in B(\lambda), \vartheta \in B(\lambda)$ the operator equation

$$
\Xi(\mathbf{v}, \mathbf{S}, \vartheta, \mathbf{k})=0
$$

has the only solution $\mathbf{v}=\mathcal{V}(\vartheta, \mathbf{k}), \mathbf{S}=\mathcal{S}(\vartheta, \mathbf{k})$, in the ball $V(\mu) \times \Sigma(\mu)$. The operators $\mathcal{V}: B(\lambda) \times B(\lambda) \rightarrow V(\mu), \mathcal{S}: B(\lambda) \times B(\lambda) \rightarrow \Sigma(\mu)$ are infinitely differentiable and $\mu=\mu(\varepsilon, \lambda) \rightarrow 0$ as $(\varepsilon, \lambda) \rightarrow(0,0)$. The norm of derivatives of the mappings $\mathcal{V}$ and $\mathcal{S}$ are bounded by constants depending only on $\Theta$, 
$\mathbf{R}, \Omega, p$ and $r$. It follows from this that this mappings satisfy inequalities (5.6)-(5.7). It remains to prove identity (5.8). We have for all $i=1,2,3$

$$
\partial_{p} T_{g, i p}(\theta, \mathbf{K}, D \mathbf{u})+\mathcal{E}_{i}(\theta, \mathbf{K}, D \mathbf{u})+f_{i}=0 \text { in } \Omega,
$$

where

$$
\theta=\Theta+\vartheta, \quad \mathbf{K}=\mathbf{R}+\mathbf{k}, \quad D \mathbf{u}=\mathbf{I}+\mathcal{S}(\vartheta, \mathbf{k})+D \mathcal{V}(\vartheta, \mathbf{k}) .
$$

Multiplying both the sides of this equality by $u_{j}$ and integrating the result by parts we obtain

$$
\int_{\Omega} f_{i} u_{j} d x+\int_{\partial \Omega} h_{i} u_{j} d s+\int_{\Omega} \mathcal{E}_{i} u_{j} d x=\int_{\Omega} T_{g, i p} \partial_{p} u_{j} d x .
$$

After the transmutation $(i, j) \rightarrow(j, i)$, we obtain

$$
\begin{array}{r}
\int_{\Omega}\left(\mathcal{E}_{i} u_{j}-\mathcal{E}_{j} u_{i}\right) d x+\int_{\Omega}\left(f_{i} u_{j}-f_{j} u_{i}\right) d x+\int_{\partial \Omega}\left(h_{i} u_{j}-h_{j} u_{i}\right) d s= \\
\int_{\Omega}\left(T_{g, i p} \partial_{p} u_{j}-T_{g, j p} \partial_{p} u_{i}\right) d x \equiv J_{i j} .
\end{array}
$$

Notice that

$$
J_{i j}=\int_{\Omega}\left(\mathbf{T}_{g}(\theta, \mathbf{K}, D \mathbf{u})(D \mathbf{u})^{\top}\right)_{i j} d x-\int_{\Omega}\left(\mathbf{T}_{g}(\theta, \mathbf{K}, D \mathbf{u})(D \mathbf{u})^{\top}\right)_{j i} d x
$$

Recalling expression (2.19) for $\mathbf{T}_{g}$, we obtain

$$
\mathbf{T}_{g}(\theta, \mathbf{K}, D \mathbf{u})(D \mathbf{u})^{\top}=\frac{1}{J_{K}} \mathbf{T}(\theta, \Phi) \mathbf{K}^{\top}(D \mathbf{u})^{\top}=\frac{1}{J_{K}} \mathbf{T}(\theta, \Phi) \Phi^{\top},
$$

where $\Phi=D \mathbf{u K}$. From this and Condition (H.2), we conclude that the matrix $\mathbf{T}_{g}(\theta, \mathbf{K}, D \mathbf{u})(D \mathbf{u})^{\top}$ is symmetric. Hence $J_{i j}=0$. Combining this result with (5.39), we obtain

$$
\int_{\Omega}\left(\mathcal{E}_{i} u_{j}-\mathcal{E}_{j} u_{i}\right) d x+\int_{\Omega}\left(f_{i} u_{j}-f_{j} u_{i}\right) d x+\int_{\partial \Omega}\left(h_{i} u_{j}-h_{j} u_{i}\right) d s=0 .
$$

Recalling that $u_{i}=x_{i}+S_{i p} x_{p}+v_{i}$ and noting that the couple $(\mathbf{f}, \mathbf{h})$ is equilibrated, we obtain

$$
\begin{array}{r}
\int_{\Omega}\left(f_{j} u_{i}-f_{i} u_{j}\right) d x+\int_{\partial \Omega}\left(h_{j} u_{j}-h_{i} u_{j}\right) d s= \\
S_{i p}\left(\int_{\Omega} x_{p} f_{j} d x+\int_{\partial \Omega} x_{p} h_{j} d s\right)-S_{j p}\left(\int_{\Omega} x_{p} f_{i} d x+\int_{\partial \Omega} x_{p} h_{i} d s\right)+ \\
\int_{\Omega}\left(f_{j} v_{i}-f_{i} v_{j}\right) d x+\int_{\partial \Omega}\left(h_{j} v_{j}-h_{i} v_{j}\right) d s=S_{i p} C_{p j}-S_{j p} C_{p i}+H_{i j}(\mathbf{v})-H_{j i}(\mathbf{v})= \\
(\mathbf{S C})_{i j}-(\mathbf{S C})_{i j}^{\top}+H_{i j}(\mathbf{v})-H_{i j}^{\top}(\mathbf{v})=0
\end{array}
$$


because of (5.18d) and (5.19). From this and (5.40) we obtain

$$
\int_{\Omega}\left(\mathcal{E}_{i} u_{j}-\mathcal{E}_{j} u_{i}\right) d x=0
$$

Recalling formulae (4.1)-(4.3) for $\mathcal{E}$ and noting that the matrix $\mathbf{E}$ is skewsymmetric, we can rewrite this equality in the form

$$
E_{i j} \int_{\Omega}\left(\varphi_{j} x_{j}+\varphi_{j} x_{i}\right) d x+\Upsilon_{i j}-\Upsilon_{j i}=0
$$

where

$$
\Upsilon_{i j}=E_{i p} \int_{\Omega} \varphi_{p}(\mathbf{S}+\mathbf{v})_{j} d x
$$

admits the estimate

$$
\left|\Upsilon_{i j}\right| \leq|\mathbf{E}|\left(|\mathbf{S}|+\|\mathbf{v}\|_{C(\Omega)}\right) \leq c|\mathbf{E}| \mu(\varepsilon, \lambda) .
$$

From this and (4.1) we obtain $|\mathbf{E}|(1-c \mu(\varepsilon, \lambda)) \leq 0$. Choosing $\varepsilon$ and $\lambda$ sufficiently small and noting that $\mu \rightarrow 0$ as $(\varepsilon, \lambda) \rightarrow 0$ we finally obtain $\mathbf{E}=0$ which along with (4.3) yields $\mathcal{E}=0$.

Corollary 1 Let all assumptions of Theorem 2 be satisfied. Then we can choose $\epsilon_{0}, \lambda_{0}$ in such a way that for every $\lambda \in\left(0, \lambda_{0}\right], \varepsilon \in\left(0, \varepsilon_{0}\right]$ and for all $\mathbf{k} \in B(\lambda), \vartheta \in B(\lambda)$ and for every $(\mathbf{f}, \mathbf{h}) \in \mathcal{F}_{c}$ with

$$
0<\|\mathbf{f}\|_{C(\Omega)}+\|\mathbf{h}\|_{C(\partial \Omega)} \leq \varepsilon,
$$

the operator

$$
\Lambda(\vartheta, \mathbf{k}) \equiv D_{\mathbf{v}, \mathbf{S}} \Xi(\mathcal{V}(\vartheta, \mathbf{k}), \mathcal{S}(\vartheta, \mathbf{k}), \vartheta, \mathbf{k}): \mathbb{X}_{p} \times \mathbb{S} \rightarrow \mathbb{Y}_{p} \times \mathbb{S}_{3}
$$

has a continuous inverse

$$
\Lambda(\vartheta, \mathbf{k})^{-1}: \mathbb{Y}_{p} \times \mathbb{S}_{3} \rightarrow \mathbb{X}_{p} \times \mathbb{S}_{3}
$$

which norm is bounded by a constant independent of $\vartheta, \mathbf{k}$. The mapping

$$
B(\lambda) \times B(\lambda) \ni(\vartheta, \mathbf{k}) \rightarrow \Lambda(\vartheta, \mathbf{k})^{-1} \in \mathcal{L}\left(\mathbb{Y}_{p} \times \mathbb{S} \rightarrow \mathbb{X}_{p} \times \mathbb{S}_{3}\right)
$$

is differentiable, and the norm of its derivative is bounded by a constant independent of $\vartheta, \mathbf{k}$, and $t$.

Proof In view of Lemma 5, the operator-valued function

$$
\begin{aligned}
& V(r) \times \Sigma(r) \times B(r) \times B(r) \ni(\mathbf{v}, \mathbf{S}, \vartheta, \mathbf{k}) \rightarrow \\
& D_{\mathbf{v}, \mathbf{S} \Xi(\mathbf{v}, \mathbf{S}, \vartheta, \mathbf{k})} \in \mathcal{L}\left(\mathbb{X}_{p} \times \mathbb{S}_{3} \rightarrow \mathbb{Y}_{p} \times \mathbb{S}_{3}\right)
\end{aligned}
$$

is infinitely differentiable, and hence it is continuous. On the other hand, Lemma 8 implies that the operator $D_{(\mathbf{v}, \mathbf{S})} \Xi(0,0,0,0)$ has a continuous inverse which norm is bounded by the constant independent of $\mathbf{f}$ and $\mathbf{h}$. Hence we can choose $\lambda^{*}>0, \mu^{*}>0$ such that for all $(\mathbf{v}, \mathbf{S}, \vartheta, \mathbf{k}) \in V\left(\mu^{*}\right) \times \Sigma\left(\mu^{*}\right) \times$ 
$B\left(\lambda^{*}\right) \times B\left(\lambda^{*}\right)$ the operator $D_{\mathbf{v}, \mathbf{S}} \Xi(\mathbf{v}, \mathbf{S}, \vartheta, \mathbf{k}): \mathbb{X}_{p} \times \mathbb{S}_{3} \rightarrow \mathbb{Y}_{p} \times \mathbb{S}_{3}$ also has a continuous inverse which norm is bounded by a constant independent of $\vartheta$, $\mathbf{k}$ and $(\mathbf{f}, \mathbf{h})$. Next, in view of Theorem 2 , the couple $(\mathcal{V}(\vartheta, \mathbf{k}), \mathcal{S}(\vartheta, \mathbf{k})$ belongs to the ball $V(\mu) \times \Sigma(\mu)$ for $(\vartheta, \mathbf{k}) \in B(\lambda) \times B(\lambda)$, where $\mu=\mu(\lambda, \varepsilon) \rightarrow 0$ as $(\lambda, \varepsilon) \rightarrow 0$. Hence, we can choose $\lambda_{0}>0$ and $\varepsilon>0$ such that $\lambda_{0} \leq \lambda^{*}$ and $\mu(\lambda, \varepsilon) \leq \mu^{*}$ for all $\lambda \in\left(0, \lambda_{0}\right]$ and all $\varepsilon \leq \varepsilon_{0}$. It remains to note that in view of Lemma 5 and Theorem 2, the mapping

$$
B(\lambda) \times B(\lambda) \ni(\vartheta, \mathbf{k}) \rightarrow \Lambda(\vartheta, \mathbf{k}) \in \mathcal{L}\left(\mathbb{X}_{p} \times \mathbb{S}_{3} \rightarrow \mathbb{Y}_{p} \times \mathbb{S}_{3}\right)
$$

is infinitely differentiable. Differentiability of $\Lambda^{-1}$ and the boundedness of its derivative follows from the identity $D \Lambda^{-1}=-\Lambda^{-1} D \Lambda \Lambda^{-1}$. This completes the proof.

\subsection{Proof of Theorem 3}

Denote by $\Xi(\mathbf{v}, \mathbf{S}, \vartheta, \mathbf{k}, t), t \in[0, T]$, the nonlinear operator defined by relations (5.18) with $(\mathbf{f}, \mathbf{h})$ replaced by $(\mathbf{f}(t), \mathbf{h}(t))$. It follows from the conditions of Theorem 3 that for every $t \in[0, T]$ the couples $(\mathbf{f}(t), \mathbf{h}(t))$ and $(\vartheta(t), \mathbf{k}(t))$ meet all requirements of Theorem 2. It follows from this theorem that for every $t \in[0, T]$, the operator equations

$$
\Xi(\mathbf{v}, \mathbf{S}, \vartheta(t), \mathbf{k}(t), t)=0
$$

has the only solution

$$
\mathbf{v}=\mathcal{V}(\vartheta, \mathbf{k}, t), \quad \mathbf{S}=\mathcal{S}(\vartheta, \mathbf{k}, t)
$$

in the ball $V(\mu) \times \Sigma(\mu)$. The operators $\mathcal{V}$ and $\mathcal{S}$ are defined by Theorem 2 . The only difference is that they depend on $t$, since $\mathbf{f}$ and $\mathbf{h}$ depend on $t$. The operators $\mathcal{V}$ and $\mathcal{S}$ meet all requirements of Theorem 2. In particular, they are infinitely differentiable with respect $\vartheta$ and $\mathbf{k}$ and their derivatives satisfy inequalities (5.6)-(5.7). Moreover, in view of Corollary 1, the operator

$$
\Lambda(\vartheta, \mathbf{k}, t) \equiv D_{\mathbf{v}, \mathbf{S}} \Xi(\mathcal{V}(\vartheta, \mathbf{k}, t), \mathcal{S}(\vartheta, \mathbf{k}, t), \vartheta, \mathbf{k}, t): \mathbb{X}_{p} \times \mathbb{S} \rightarrow \mathbb{Y}_{p} \times \mathbb{S}_{3}
$$

has a continuous inverse

$$
\Lambda(\vartheta, \mathbf{k}, t)^{-1}: \mathbb{Y}_{p} \times \mathbb{S}_{3} \rightarrow \mathbb{X}_{p} \times \mathbb{S}_{3}
$$

which norm is bounded by a constant independent of $\vartheta, \mathbf{k}$. The mapping

$$
B(\lambda) \times B(\lambda) \ni(\vartheta, \mathbf{k}) \rightarrow \Lambda(\vartheta, \mathbf{k}, t)^{-1} \in \mathcal{L}\left(\mathbb{Y}_{p} \times \mathbb{S}_{3} \rightarrow \mathbb{X}_{p} \times \mathbb{S}_{3}\right)
$$

is differentiable, and the norm of its derivative is bounded by a constant independent of $\vartheta, \mathbf{k}$, and $t$.

Hence Theorem 3 will be proved if we show that the time derivative of Id $+\mathbf{S}+\mathbf{v}$ admits the representation (5.14) with operators $\mathcal{P}, \mathcal{Q}_{i}$ satisfying 
inequalities (5.15). In order to do so we rewrite operator equation (5.44) in the form

$$
\begin{array}{r}
\Xi_{0}(\mathbf{v}, \mathbf{S}, \vartheta(t), \mathbf{k}(t), t)=0, \quad \Xi_{b}(\mathbf{v}, \mathbf{S}, \vartheta(t), \mathbf{k}(t), t)=0, \\
\mathbf{S C}-(\mathbf{S C})^{\top}+\mathbf{H}(\mathbf{v})-\mathbf{H}(\mathbf{v})^{\top}=0 .
\end{array}
$$

Differentiating both sides of these equalities by $t$ and recalling expression (5.18) for the operator $\Xi$, we obtain

$$
\Lambda(\vartheta, \mathbf{k}, t)\left[\partial_{t} \mathbf{v}, \partial_{t} \mathbf{S}\right]=\mathbf{G}+\mathcal{T}_{1}(\vartheta, \mathbf{k})\left[\partial_{t} \vartheta\right]+\mathcal{T}_{2}(\vartheta, \mathbf{k})\left[\partial_{t} \mathbf{k}\right]=0 .
$$

where the linear operators $\mathcal{T}_{i}$ are defined by the equalities

$\mathcal{T}_{1}(\vartheta, \mathbf{k})=D_{\vartheta} \Xi(\mathcal{V}(\vartheta, \mathbf{k}, t), \mathcal{S}(\vartheta, \mathbf{k}, t), \vartheta, \mathbf{k}, t), \quad \mathcal{T}_{2}(\vartheta, \mathbf{k})=D_{\mathbf{k}} \Xi(\mathcal{V}(\vartheta, \mathbf{k}, t), \mathcal{S}(\vartheta, \mathbf{k}, t), \vartheta, \mathbf{k}, t)$.

and vector valued function $\mathbf{G}$ is defined by

$$
\mathbf{G}=\left(\partial_{t} \mathbf{f}, \partial_{t} \mathbf{h}, \frac{1}{\|\mathbf{f}\|_{C(\Omega)}+\|\mathbf{h}\|_{C(\partial \Omega)}}\left\{\mathbf{S C}_{t}-\left(\mathbf{S C}_{t}\right)^{\top}+\mathbf{H}_{t}(\mathbf{v})-\mathbf{H}_{t}(\mathbf{v})^{\top}\right\}\right) .
$$

Here $\mathbf{v}=\mathcal{V}(\vartheta(t), \mathbf{k}(t), t), \quad \mathbf{S}=\mathcal{S}(\vartheta(t), \mathbf{k}(t), t)$, the matrix $\mathbf{C}_{t}$ and the integral operator $\mathbf{H}_{t}$ are defined by formulae (4.5i) with $\mathbf{f}$ and $\mathbf{h}$ replaced by $\partial_{t} \mathbf{f}$ and $\partial_{t} \mathbf{h}$.

Since for every $t \in[0, T]$ the mapping

$$
V(r) \times \Sigma(r) \times B(r) \times B(r) \ni(\mathbf{v}, \mathbf{S}, \vartheta, \mathbf{k}) \rightarrow \Xi(\mathbf{v}, \mathbf{S}, \vartheta, \mathbf{k}) \in \mathbb{Y}_{p} \times \mathbb{S}_{3}
$$

is infinitely differentiable, the mappings

$$
B(\lambda) \times B(\lambda) \ni(\vartheta, \mathbf{k}) \rightarrow \mathcal{T}_{i}(\vartheta, \mathbf{k}, t) \in \mathcal{L}\left(W^{1, p}(\Omega) \rightarrow \mathbb{Y}_{p} \times \mathbb{S}_{3}\right)
$$

are differentiable, and the norms of their derivatives are bounded by a constant independent of $\vartheta, \mathbf{k}$, and $t$. Thus we get

$$
\left(\partial_{t} \mathbf{v}, \partial_{t} \mathbf{S}\right)=-\Lambda(\vartheta, \mathbf{k}, t)^{-1}\left(\mathbf{G}+\mathcal{T}_{1}(\vartheta, \mathbf{k}, t)\left[\partial_{t} \vartheta\right]+\mathcal{T}_{2}(\vartheta, \mathbf{k}, t)\left[\partial_{t} \mathbf{k}\right]\right) .
$$

Now introduce the projections

$$
\Pi_{1}:\left[\partial_{t} \mathbf{v}, \partial_{t} \mathbf{S}\right] \rightarrow \partial_{t} \mathbf{v}, \quad \Pi_{2}:\left[\partial_{t} \mathbf{v}, \partial_{t} \mathbf{S}\right] \rightarrow \partial_{t} \mathbf{S} .
$$

Noting that

$$
\partial_{t} \mathbf{u}=\partial_{t} \mathbf{S} x+\partial_{t} \mathbf{v}
$$

we obtain the desired representation

$$
\partial_{t} \mathbf{u}=\mathcal{P}(\vartheta, \mathbf{k}, t)+\mathcal{Q}_{1}(\vartheta, \mathbf{k}, t) \partial_{t} \vartheta+\mathcal{Q}_{2}(\vartheta, \mathbf{k}, t) \partial_{t} \mathbf{k} .
$$

with vector-valued function $\mathcal{P}$ and the linear operators $\mathcal{Q}_{i}$ defined by

$$
\begin{gathered}
\mathcal{P}=-\Pi_{2}\left(\Lambda^{-1} \mathbf{G}\right) x-\Pi_{1} \Lambda^{-1} \mathbf{G} \\
\mathcal{Q}_{1}\left[\partial_{t} \vartheta\right]=-\Pi_{2}\left(\Lambda^{-1} \mathcal{T}_{1}\left[\partial_{t} \vartheta\right]\right) x-\Pi_{1} \Lambda^{-1} \mathcal{T}_{1}\left[\partial_{t} \vartheta\right] \\
\mathcal{Q}_{2}\left[\partial_{t} \mathbf{k}\right]=-\Pi_{2}\left(\Lambda^{-1} \mathcal{T}_{2}\left[\partial_{t} \mathbf{k}\right]\right) x-\Pi_{1} \Lambda^{-1} \mathcal{T}_{2}\left[\partial_{t} \mathbf{k}\right]
\end{gathered}
$$


where

$$
\Lambda=\Lambda(\vartheta, \mathbf{k}, t), \quad \mathcal{T}_{i}=\mathcal{T}_{i}(\vartheta, \mathbf{k}, t) .
$$

Notice that the mappings

$$
\begin{gathered}
B(\lambda) \times B(\lambda) \ni(\vartheta, \mathbf{k}) \rightarrow \mathcal{P}(\vartheta, \mathbf{k}, t) \in W^{1, p}(\Omega), \\
B(\lambda) \times B(\lambda) \ni(\vartheta, \mathbf{k}) \rightarrow \mathcal{Q}_{i}(\vartheta, \mathbf{k}, t) \in \mathcal{L}\left(W^{1, p}(\Omega) \rightarrow W^{2, p}(\Omega)\right)
\end{gathered}
$$

are continuously differentiable as products of infinitely differentiable mappings. Moreover, the norms of their derivatives are bounded by a constant independent on $\vartheta$, $\mathbf{k}$ and $t$. Hence these mappings satisfy the Lipschitz condition in $B(\lambda) \times B(\lambda)$ uniformly in $t$, which yields dthe esired estimates (5.15)-(5.16). It remains to prove the estimate (5.17). To this end, notice that the operator $\Lambda^{-1}: \mathbb{Y}_{p} \times \mathbb{S}_{3} \rightarrow \mathbb{X}_{p} \times \mathbb{S}_{3}$ is bounded and $\mathbb{Y}$ is continuously embedded in $W^{2, p}(\Omega)$. From this and conditions (5.10)-(5.11), we finally obtain

$$
\begin{gathered}
\|\mathcal{P}\|_{W^{2, p}(\Omega)} \leq c\|\mathbf{G}\|_{L^{p}(\Omega)} \leq c\left(\left\|\partial_{t} \mathbf{f}(t)\right\|_{C(\Omega)}+\left\|\partial_{t} \mathbf{h}(t)\right\|_{C(\partial \Omega)}\right)+ \\
\frac{\left\|\partial_{t} \mathbf{f}(t)\right\|_{C(\Omega)}+\left\|\partial_{t} \mathbf{h}(t)\right\|_{C(\partial \Omega)}}{\|\mathbf{f}(t)\|_{C(\Omega)}+\|\mathbf{h}(t)\|_{C(\partial \Omega)}}\left(|\mathbf{S}|+\|\mathbf{v}\|_{L^{1}(\Omega)}\right) \leq \\
c \varepsilon+c|\mathbf{S}|+c\|\mathbf{v}\|_{L^{1}(\Omega)} \leq c(\varepsilon+\lambda),
\end{gathered}
$$

and the theorem follows.

\section{Proof of Theorem 1}

We are now in a position to prove the main Theorem 1 on solvability of the traction boundary value problem (3.5)-(3.6). First we prove the local existence and uniqueness of solutions to the modified boundary value problem (4.5). Next we show that solutions to the modified problem serve as solutions to problem (3.5)-(3.6). In order to prove the solvability of the modified problem (4.5), we employ Theorem 3. Using this theorem, we eliminate $\mathbf{u}$ from equations (4.5) and reduce the modified problem to the system of evolutionary operator equations. To do this, it is convenient to introduce the following operators

$$
\begin{gathered}
\mathcal{N}_{0}=-\kappa \chi \Delta \frac{1}{\Theta} \\
\mathcal{N}_{1}(\vartheta, \mathbf{k})=\chi\left(c_{T}(\Theta+\vartheta) \frac{1}{J_{K}^{2}} \partial_{t} J_{K}-c_{T}\left(\frac{1}{J_{K}}-1\right) \partial_{t} \vartheta\right) \\
\mathcal{N}_{2}(\vartheta, \mathbf{k})=-\kappa \chi \Delta\left(\frac{\vartheta^{2}}{\Theta^{2}(\Theta+\vartheta)}\right) \\
\mathcal{M}(\vartheta, \mathbf{k})=\chi \mathbf{T}_{g}(\Theta+\vartheta, \mathbf{R}+\mathbf{k}, D \mathbf{u}) \cdot D \partial_{t} \mathbf{u} \\
\mathcal{G}(\vartheta, \mathbf{k})=\mathbf{g}(\vartheta, \mathbf{R}+\mathbf{k}, D \mathbf{u})
\end{gathered}
$$


Here $\chi(t)$ is given by (4.4), and $D \mathbf{u}, \partial_{t} \mathbf{u}$ are given by

$$
\begin{array}{r}
D \mathbf{u}=\mathbf{I}+\mathcal{S}(\vartheta, \mathbf{k}, t)+D \mathcal{V}(\vartheta, \mathbf{k}, t), \\
\partial_{t} \mathbf{u}=\mathcal{P}(\vartheta, \mathbf{k}, t)+\mathcal{Q}_{1}(\vartheta, \mathbf{k}, t)\left[\partial_{t} \vartheta\right]+\mathcal{Q}_{2}(\vartheta, \mathbf{k}, t)\left[\partial_{t} \mathbf{k}\right],
\end{array}
$$

where the operators $\mathcal{S}, \mathcal{V}, \mathcal{P}$, and $\mathcal{Q}_{i}$ are defined by Theorem 3 . Denote by $Q$ the cylinder $Q=\Omega \times(0, T)$. Using this notation and recalling Theorem 3 , we can rewrite problem 4.5 in the form

$$
\begin{gathered}
c_{T} \frac{\partial \vartheta}{\partial t}-\kappa \Delta\left(\frac{\vartheta}{\Theta^{2}}\right)=\mathcal{N}_{0}+\sum_{1}^{2} \mathcal{N}_{i}(\vartheta, \mathbf{k})+\mathcal{M}(\vartheta, \mathbf{k}) \text { in } Q \\
\frac{\partial \mathbf{k}}{\partial t}=\chi \mathcal{G}(\vartheta, \mathbf{k}) \text { in } Q \\
\partial_{n} \vartheta=0 \text { on } \partial \Omega \times(0, T) \\
\vartheta(x, 0)=0, \quad \mathbf{k}(x, 0)=\mathbf{k}_{0}(x) \text { in } \Omega .
\end{gathered}
$$

We will consider problem (6.2) in the Banach spaces $\mathbb{E}_{p}$ and $\mathbb{F}_{p}$ given by the following definition

Definition 7 For every $p \in(1, \infty)$ denote by $\mathbb{E}_{p}$ and $\mathbb{F}_{p}$ the Banach spaces which consist respectively of all functions $\vartheta: \Omega \times[0, T] \rightarrow \mathbb{R}$ and $k: \Omega \times$ $[0, T] \rightarrow \mathbb{R}$ with finite norms

$$
\begin{gathered}
\|\vartheta\|_{\mathbb{E}_{p}}=\left\|\partial_{t} \vartheta\right\|_{L^{p}\left(0, T ; W^{1, p}(\Omega)\right)}+\|\vartheta\|_{L^{p}\left(0, T ; W^{3, p}(\Omega)\right)}+\|\vartheta\|_{L^{\infty}\left(0, T ; W^{2, p}(\Omega)\right)}, \\
\|k\|_{\mathbb{F}_{p}}=\left\|\partial_{t} k\right\|_{L^{p}\left(0, T ; W^{1, p}(\Omega)\right)}+\|k\|_{L^{\infty}\left(0, T ; W^{1, p}(\Omega)\right)} .
\end{gathered}
$$

The same notation is used for the spaces of vector-valued and matrix-valued functions.

Notice that for $p>3$, the spaces $\mathbb{E}_{p}$ and $\mathbb{F}_{p}$ are Banach algebras, i. e., there is $c$ depending only on $\Omega, T$ and $p$ such that the inequalities

$$
\|\vartheta h\|_{\mathbb{E}_{p}} \leq c\|\vartheta\|_{\mathbb{E}_{p}}\|h\|_{\mathbb{E}_{p}}, \quad\|f k\|_{\mathbb{F}_{p}} \leq c\|f\|_{\mathbb{F}_{p}}\|k\|_{\mathbb{F}_{p}}
$$

hold for all $\vartheta, h \in \mathbb{E}_{p}$ and all $f, k \in \mathbb{F}_{p}$.

Now our task is to prove the local solvability of problem (6.2) in the space $\mathbb{E}_{p} \times \mathbb{F}_{p}$. We split the proof into a sequence of lemmas. The first technical lemma gives the estimates for the inverse temperature and will be used throughout the proof.

Lemma 9 Let $p>3$. Then there are $\rho_{0}>0$ and $c>0$, depending only on $p$, $\Omega, \mathbf{R}$ and $\Theta$, such that

$$
\begin{gathered}
\left\|(\Theta+\vartheta)^{-1}\right\|_{\mathbb{E}_{p}} \leq c . \\
0<c^{-1}<\Theta+\vartheta<c, \quad|\mathbf{k}| \leq 1 / 2 \\
|\vartheta|<c \varrho, \quad|\mathbf{k}| \leq c \rho .
\end{gathered}
$$

for $\operatorname{all}(\vartheta, \mathbf{k}) \in \Gamma\left(\rho_{0}\right), \rho \in\left(0, \rho_{0}\right)$. 
Proof We have

$$
(\Theta+\vartheta)^{-1}=\Theta^{-1} \sum_{k \geq 0}\left(\Theta^{-1} \vartheta\right)^{k} .
$$

Since $\Theta \in C^{\infty}(\Omega)$ is strictly positive function and $\mathbb{E}_{p}$ is a Banach algebra, we have

$$
\left\|(\Theta+\vartheta)^{-1}\right\|_{\mathbb{E}_{p}} \leq c \sum_{k \geq 0} c^{k}\|\vartheta\|_{\mathbb{E}_{p}}^{k}
$$

Choosing $\varrho_{0} \leq 1 / c$, we arrive at (6.3). Inequality (6.4) follows from the boundedness of the embedding $\mathbb{E}_{p}, \mathbb{F}_{p} \hookrightarrow L^{\infty}(Q)$.

The next lemma establishes the properties of the operators $\mathcal{N}_{i}$. For every $\rho>0$, let denote by $\Gamma(\rho) \subset \mathbb{E}_{p} \times \mathbb{F}_{p}$ the ball of radius $\rho$ centered at 0 ,

$$
\Gamma(\rho)=\left\{(\vartheta, \mathbf{k}) \in \mathbb{E}_{p} \times \mathbb{F}_{p}: \max \left\{\|\vartheta\|_{\mathbb{E}_{p}},\|\mathbf{k}\|_{\mathbb{F}_{p}}\right\} \leq \rho\right\} .
$$

Lemma 10 Let $p>3$. Then there are $\rho_{0}>0$ and $c>0$, depending only on $p, \Omega, \mathbf{R}$ and $\Theta$, with the following properties. For every $\rho \in\left(0, \rho_{0}\right]$ and $\left(\vartheta_{j}, \mathbf{k}_{j}\right) \in \Gamma(\rho), j=1,2$,

$$
\begin{gathered}
\left\|\mathcal{N}_{i}\left(\vartheta_{1}, \mathbf{k}_{1}\right)-\mathcal{N}_{i}\left(\vartheta_{2}, \mathbf{k}_{2}\right)\right\|_{L^{p}\left(0, T ; W^{1, p}(\Omega)\right)} \leq c \rho\left\|\vartheta_{1}-\vartheta_{2}\right\|_{\mathbb{E}_{p}}+c\left\|\mathbf{k}_{1}-\mathbf{k}_{2}\right\|_{\mathbb{F}_{p}} . \\
\left\|\mathcal{N}_{i}\left(\vartheta_{1}, \mathbf{k}_{1}\right)\right\|_{\left.L^{p}\left(0, T ; W^{1, p}(\Omega)\right)\right)} \leq c \rho \text { for } i=1,2 \\
\left\|\mathcal{N}_{0}\right\|_{\left.L^{p}\left(0, T ; W^{1, p}(\Omega)\right)\right)} \leq c T_{0}^{1 / p}
\end{gathered}
$$

Proof Let $\rho_{0}$ be given by Lemma 9 , and choose an arbitrary $\rho \in\left(0, \rho_{0}\right]$ and $\left(\vartheta_{1}, \mathbf{k}_{1}\right),\left(\vartheta_{2}, \mathbf{k}_{2}\right) \in \Gamma(\rho)$. Since $W^{1, p}(\Omega), p>3$, is a Banach algebra, inequalities (6.4), (6.5), and the identity $J_{K}=\operatorname{det}\left(\mathbf{I}+\mathbf{R}^{\top} \mathbf{k}\right)$ imply the estimates

$$
\begin{array}{r}
\left\|J_{K_{1}}^{-1}-J_{K_{2}}^{-1}\right\|_{W^{1, p}(\Omega)} \leq c\left\|\mathbf{k}_{1}-\mathbf{k}_{2}\right\|_{W^{1, p}(\Omega)}, \\
\left\|J_{K_{1}}^{-1}-1\right\|_{W^{1, p}(\Omega)}+\mid J_{K_{2}}^{-1}-1 \|_{W^{1, p}(\Omega)} \leq c\left(\left\|\mathbf{k}_{1}\right\|_{W^{1, p}(\Omega)}+\left\|\mathbf{k}_{2}\right\|_{W^{1, p}(\Omega)}\right) \leq c \rho \\
\left\|\partial_{t}\left(J_{K_{1}}^{-1}-J_{K_{2}}^{-1}\right)\right\|_{W^{1, p}(\Omega)} \leq c\left\|\partial_{t} \mathbf{k}_{1}-\partial_{t} \mathbf{k}_{2}\right\|_{W^{1, p}(\Omega)}+\left(\left\|\partial_{t} \mathbf{k}_{1}\right\|_{W^{1, p}(\Omega)}+\right. \\
\left.\left\|\partial_{t} \mathbf{k}_{2}\right\|_{W^{1, p}(\Omega)}\right)\left\|\mathbf{k}_{1}-\mathbf{k}_{2}\right\|_{W^{1, p}(\Omega)} .
\end{array}
$$

From this and (6.1b), we obtain

$$
\begin{gathered}
\left\|\mathcal{N}_{1}\left(\vartheta_{1}, \mathbf{k}_{1}\right)-\mathcal{N}_{1}\left(\vartheta_{2}, \mathbf{k}_{2}\right)\right\|_{W^{1, p}(\Omega)} \leq c\left\|\partial_{t} \mathbf{k}_{1}-\partial_{t} \mathbf{k}_{2}\right\|_{W^{1, p}(\Omega)}+ \\
c\left(\left\|\partial_{t} \mathbf{k}_{1}\right\|_{W^{1, p}(\Omega)}+\left\|\partial_{t} \mathbf{k}_{2}\right\|_{W^{1, p}(\Omega)}\right)\left\|\mathbf{k}_{1}-\mathbf{k}_{2}\right\|_{W^{1, p}(\Omega)}+ \\
c \rho\left\|\partial_{t}\left(\vartheta_{1}-\vartheta_{2}\right)\right\|_{W^{1, p}(\Omega)}+c\left(\left\|\mathbf{k}_{1}-\mathbf{k}_{2}\right\|_{W^{1, p}(\Omega)}\right)\left(\left|\partial_{t} \vartheta_{1}\right|+\left|\partial_{t} \vartheta_{2}\right|\right) \leq c\left\|\partial_{t} \mathbf{k}_{1}-\partial_{t} \mathbf{k}_{2}\right\|_{W^{1, p}(\Omega)}+ \\
c\left(\left\|\partial_{t} \mathbf{k}_{1}\right\|_{W^{1, p}(\Omega)}+\left\|\partial_{t} \mathbf{k}_{2}\right\|_{W^{1, p}(\Omega)}\right)\left\|\mathbf{k}_{1}-\mathbf{k}_{2}\right\|_{W^{1, p}(\Omega)}+c \rho\left\|\partial_{t}\left(\vartheta_{1}-\vartheta_{2}\right)\right\|_{W^{1, p}(\Omega)}+ \\
c\left(\left\|\mathbf{k}_{1}-\mathbf{k}_{2}\right\|_{W^{1, p}(\Omega)}\right)\left(\left\|\partial_{t} \vartheta_{1}\right\|_{W^{1, p}(\Omega)}+\left\|\partial_{t} \vartheta_{2}\right\|_{W^{1, p}(\Omega)}\right) .
\end{gathered}
$$

This leads to the inequality

$$
\begin{gathered}
\left\|\mathcal{N}_{1}\left(\vartheta_{1}, \mathbf{k}_{1}\right)-\mathcal{N}_{1}\left(\vartheta_{2}, \mathbf{k}_{2}\right)\right\|_{L^{p}\left(0, T ; W^{1, p}(\Omega)\right)} \leq\left\|\partial_{t} \mathbf{k}_{1}-\partial_{t} \mathbf{k}_{2}\right\|_{L^{p}\left(0, T ; W^{1, p}(\Omega)\right)}+ \\
\left\|\mathbf{k}_{1}-\mathbf{k}_{2}\right\|_{L^{\infty}\left(0, T ; W^{1, p}(\Omega)\right)}\left(\left\|\partial_{t} \mathbf{k}_{1}\right\|_{L^{p}\left(0, T ; W^{1, p}(\Omega)\right)}+\left\|\partial_{t} \mathbf{k}_{2}\right\|_{L^{p}\left(0, T ; W^{1, p}(\Omega)\right)}\right)+ \\
c \rho\left\|\partial_{t}\left(\vartheta_{1}-\vartheta_{2}\right)\right\|_{L^{p}\left(0, T ; W^{1, p}(\Omega)\right)}+c\left\|\mathbf{k}_{1}-\mathbf{k}_{2}\right\|_{L^{\infty}\left(0, T ; W^{1, p}(\Omega)\right)}\left(\left\|\partial_{t} \vartheta_{1}\right\|_{L^{p}\left(0, T ; W^{1, p}(\Omega)\right)}+\right. \\
\left.\left\|\partial_{t} \vartheta_{2}\right\|_{L^{p}\left(0, T ; W^{1, p}(\Omega)\right)}\right) \leq\left\|\mathbf{k}_{1}-\mathbf{k}_{2}\right\|_{\mathbb{F}_{p}}+c \rho\left\|\vartheta_{1}-\vartheta_{2}\right\|_{\mathbb{E}_{p}},
\end{gathered}
$$


which yields estimate (6.6) for $\mathcal{N}_{1}$. The estimate $\mathcal{N}_{3}$ is similar to the estimate of $\mathcal{N}_{2}$. We have

$$
\begin{gathered}
\left\|\mathcal{N}_{3}\left(\vartheta_{1}\right)-\mathcal{N}_{3}\left(\vartheta_{2}\right)\right\|_{L^{p}(Q)} \leq c\left\|\frac{\vartheta_{1}^{2}}{\Theta+\vartheta_{1}}-\frac{\vartheta_{2}^{2}}{\Theta+\vartheta_{2}}\right\|_{\mathbb{E}_{p}}= \\
\left\|\frac{\left(\vartheta_{1}-\vartheta_{2}\right)\left(\Theta\left(\vartheta_{1}+\vartheta_{2}\right)+\vartheta_{1} \vartheta_{2}\right)}{\left(\Theta+\vartheta_{1}\right)\left(\Theta+\vartheta_{2}\right)}\right\|_{\mathbb{E}_{p}} \leq \\
c\left\|\vartheta_{1}-\vartheta_{2}\right\|_{\mathbb{E}_{p}}\left(\left\|\vartheta_{1}\right\|_{\mathbb{E}_{p}}\left\|\vartheta_{2}\right\|_{\mathbb{E}_{p}}+\left\|\vartheta_{1} \vartheta_{2}\right\|_{\mathbb{E}_{p}}\right)\left\|\frac{1}{\Theta+\vartheta_{1}}\right\|_{\mathbb{E}_{p}}\left\|\frac{1}{\Theta+\vartheta_{2}}\right\|_{\mathbb{E}_{p}} \leq \\
c \rho\left\|\vartheta_{1}-\vartheta_{2}\right\|_{\mathbb{E}_{p}},
\end{gathered}
$$

which yields the estimate (6.6) for $\mathcal{N}_{3}$. Notice that $\mathcal{N}_{i}(0,0)=0$ for $i=1,2,3$. Setting $\left(\vartheta_{2}, \mathbf{k}_{2}\right)=0$ and applying $(6.6)$, we obtain $(6.7)$. It remains to note that

$$
\left\|\mathcal{N}_{0}\right\|_{L^{p}\left(0, T ; W^{1, p}(\Omega)\right)} \leq c\|\chi\|_{L^{p}\left(0, T_{0}\right)} \leq c T_{0}^{1 / p} .
$$

Lemma 11 Under the assumptions of Theorem 1, there are $\rho_{0}>0, \varepsilon>0$, and $c>0$, depending only on $p>3, \Omega, T, \mathbf{R}$ and $\Theta$, with the following properties. For every $\rho \in\left(0, \rho_{0}\right]$ and $\left(\vartheta_{1}, \mathbf{k}_{1}\right),\left(\vartheta_{1}, \mathbf{k}_{1}\right) \in \Gamma(\rho)$,

$$
\begin{gathered}
\left\|\mathcal{M}\left(\vartheta_{1}, \mathbf{k}_{1}\right)-\mathcal{M}\left(\vartheta_{2}, \mathbf{k}_{2}\right)\right\|_{L^{p}\left(0, T ; W^{1, p}(\Omega)\right)} \leq \\
c(\rho+\varepsilon)\left(\left\|\vartheta_{1}-\vartheta_{2}\right\|_{\mathbb{E}_{p}}+\left\|\mathbf{k}_{1}-\mathbf{k}_{2}\right\|_{\mathbb{F}_{p}}\right) \\
\left\|\mathcal{M}\left(\vartheta_{i}, \mathbf{k}_{i}\right)\right\|_{L^{p}\left(0, T ; W^{1, p}(\Omega)\right)} \leq c \rho(\varepsilon+\rho) \\
\left\|\mathcal{G}\left(\vartheta_{1}, \mathbf{k}_{1}\right)-\mathcal{G}\left(\vartheta_{2}, \mathbf{k}_{2}\right)\right\|_{L^{\infty}\left(0, T ; W^{1, p}(\Omega)\right)} \leq c\left\|\vartheta_{1}-\vartheta_{2}\right\|_{\mathbb{E}_{p}}+c\left\|\mathbf{k}_{1}-\mathbf{k}_{2}\right\|_{\mathbb{F}_{p}} \\
\left\|\mathcal{G}\left(\vartheta_{1}, \mathbf{k}_{1}\right)\right\|_{L^{\infty}\left(0, T ; W^{1, p}(\Omega)\right)} \leq c
\end{gathered}
$$

Proof Since $\mathcal{G}$ is independent of $\partial_{t} \mathbf{u}$ and $\mathbf{g}$ is infinitely differentiable, inequalities (6.11) and (6.12) are simpler than (6.9) and (6.10). In order to avoid repetitions, we prove $(6.9)$ and $(6.10)$ only. We have for a.e. $t \in(0, T)$,

$$
\begin{array}{r}
\left\|\mathcal{M}\left(\vartheta_{1}, \mathbf{k}_{1}\right)-\mathcal{M}\left(\vartheta_{2}, \mathbf{k}_{2}\right)\right\|_{W^{1, p}(\Omega)} \leq \\
\left\|\mathbf{T}\left(\theta_{1}, \mathbf{K}_{1}, D \mathbf{u}_{1}\right)-\mathbf{T}\left(\theta_{2}, \mathbf{K}_{2}, D \mathbf{u}_{2}\right)\right\|_{W^{1, p}(\Omega)}\left(\left\|D \partial_{t} \mathbf{u}_{1}\right\|_{W^{1, p}(\Omega)}+\left\|D \partial_{t} \mathbf{u}_{2}\right\|_{W^{1, p}(\Omega)}\right)+ \\
\left(\left\|\mathbf{T}\left(\theta_{1}, \mathbf{K}_{1}, D \mathbf{u}_{1}\right)\right\|_{W^{1, p}(\Omega)}+\left|\mathbf{T}\left(\theta_{2}, \mathbf{K}_{2}, D \mathbf{u}_{2}\right)\right|\right)\left\|D \partial_{t} \mathbf{u}_{1}-D \partial_{t} \mathbf{u}_{2}\right\|_{W^{1, p}(\Omega)},
\end{array}
$$

where

$$
\begin{array}{r}
D \mathbf{u}_{j}=\mathbf{I}+\mathcal{S}\left(\vartheta_{j}, \mathbf{k}_{j}, t\right)+D \mathcal{V}\left(\vartheta_{j}, \mathbf{k}_{j}, t\right), \\
\partial_{t} \mathbf{u}_{j}=D \mathcal{P}\left(\vartheta_{j}, \mathbf{k}_{j}, t\right)+\mathcal{Q}_{1}\left(\vartheta_{j}, \mathbf{k}_{j}, t\right)\left[\partial_{t} \vartheta_{j}\right]+\mathcal{Q}_{2}\left(\vartheta_{j}, \mathbf{k}_{j}, t\right)\left[\partial_{t} \mathbf{k}_{j}\right] .
\end{array}
$$

Now choose $\varrho_{0} \leq \lambda$, where $\lambda$ is given by Theorem 3 . Assume that $\varepsilon_{0}$ is given by this theorem. It follows from this Theorem and Definition 7 of Banach spaces $\mathbb{E}_{p}$ and $\mathbf{F}_{p}$ that the $\mathcal{S}(\vartheta(t), \mathbf{k}(t), t)$ and $\mathcal{V}(\vartheta(t), \mathbf{k}(t), t)$ are well defined 
for $(\vartheta, \mathbf{k}) \in \Gamma(\rho)$, for $\rho \leq \rho_{0}, \varepsilon \leq \varepsilon_{0}$, and for a. e. $t \in[0, T]$. In particular, they satisfy inequalities (5.6) and (5.7), which yield

$$
\begin{array}{r}
\|D \mathcal{V}(0,0, t)\|_{W^{1, p}(\Omega)}+|\mathcal{S}(0,0, t)| \leq c\|\mathcal{V}(0,0, t)\|_{\mathbb{X}_{p}}+c|\mathcal{S}(0,0, t)| \leq c \varepsilon, \\
\left\|D \mathcal{V}\left(\vartheta_{1}, \mathbf{k}_{1}, t\right)-D \mathcal{V}\left(\vartheta_{2}, \mathbf{k}_{2}, t\right)\right\|_{W^{1, p}(\Omega)}+\left|\mathcal{S}\left(\vartheta_{1}, \mathbf{k}_{1}, t\right)-\mathcal{S}\left(\vartheta_{2}, \mathbf{k}_{2}, t\right)\right| \leq \\
c\left\|\mathcal{V}\left(\vartheta_{1}, \mathbf{k}_{1}, t\right)-\mathcal{V}\left(\vartheta_{2}, \mathbf{k}_{2}, t\right)\right\|_{\mathbb{X}_{p}}+\left|\mathcal{S}\left(\vartheta_{1}, \mathbf{k}_{1}, t\right)-\mathcal{S}\left(\vartheta_{2}, \mathbf{k}_{2}, t\right)\right| \leq \\
c\left(\left\|\vartheta_{1}(t)-\vartheta_{2}(t)\right\|_{W^{2, p}(\Omega)}+\left\|\mathbf{k}_{1}(t)-\mathbf{k}_{2}(t)\right\|_{W^{1, p}(\Omega)}\right) \leq \\
c\left(\left\|\vartheta_{1}-\vartheta_{2}\right\|_{\mathbb{E}_{p}}+\left\|\mathbf{k}_{1}-\mathbf{k}_{2}\right\|_{\mathbb{F}_{p}}\right) .
\end{array}
$$

Setting $\left(\vartheta_{1}, \mathbf{k}_{1}\right)=0$ and next $\left(\vartheta_{2}, \mathbf{k}_{2}\right)=0$, we get

$$
\left\|D \mathcal{V}\left(\vartheta_{j}, \mathbf{k}_{j}, t\right)\right\|_{W^{1, p}(\Omega)}+\left|\mathcal{S}\left(\vartheta_{j}, \mathbf{k}_{j}, t\right)\right| \leq c \varepsilon+c \rho .
$$

Next, we have

$$
\mathbf{T}_{g}(\theta, \mathbf{K}, D \mathbf{u})=\frac{\Theta+\vartheta}{\operatorname{det}\left(\mathbf{I}+\mathbf{R}^{\top} \mathbf{k}\right)} \frac{\partial W}{\partial \Phi}((\mathbf{I}+\mathcal{S}+D \mathcal{V})(\mathbf{R}+\mathbf{k}))(\mathbf{R}+\mathbf{k})^{\top} .
$$

The right hand side can be regarded as a function of the entries of matrices $\mathbf{R}$ $\mathbf{k}, \mathcal{S}, D \mathcal{V}$. It follows from Condition $(\mathbf{H} .1)$ that there is $r>0$ such that this function is infinitely differentiable for $|\mathbf{k}| \leq r,|D \mathcal{V}| \leq r$, and $|\mathcal{S}| \leq r$. Now choose $\varepsilon_{0}$ and $\rho_{0}$ small enough that $2 c \varrho_{0}<r, 2 c \varepsilon_{0} \leq r$. It follows from this and (6.16) that for a.e. $t \in(0, T)$,

$$
\begin{array}{r}
\left\|\mathbf{T}\left(\theta_{1}, \mathbf{K}_{1}, D \mathbf{u}_{1}\right)(t)-\mathbf{T}\left(\theta_{2}, \mathbf{K}_{2}, D \mathbf{u}_{2}\right)(t)\right\|_{W^{1, p}(\Omega)} \leq \\
c\left(\left\|\vartheta_{1}(t)-\vartheta_{2}(t)\right\|_{W^{1, p}(\Omega)}+\left\|\mathbf{k}_{1}(t)-\mathbf{k}_{2}(t)\right\|_{W^{1, p}(\Omega)}+\right. \\
\left\|D \mathcal{V}\left(\vartheta_{1}, \mathbf{k}_{1}, t\right)-D \mathcal{V}\left(\vartheta_{2}, \mathbf{k}_{2}, t\right)\right\|_{W^{1, p}(\Omega)}+ \\
\left|\mathcal{S}\left(\vartheta_{1}, \mathbf{k}_{1}, t\right)-\mathcal{S}\left(\vartheta_{2}, \mathbf{k}_{2}, t\right)\right| \leq c\left(\left\|\vartheta_{1}-\vartheta_{2}\right\|_{\mathbb{E}_{p}}+\left\|\mathbf{k}_{1}-\mathbf{k}_{2}\right\|_{\mathbb{F}_{p}}\right) .
\end{array}
$$

Notice that $\mathbf{T}_{g}(\Theta, \mathbf{R}, \mathbf{I})=0$. From this and (6.17), we obtain

$$
\begin{array}{r}
\left\|\mathbf{T}\left(\theta_{j}, \mathbf{K}_{j}, D \mathbf{u}_{j}\right)(t)\right\|_{W^{1, p}(\Omega)} \\
\left\|\mathbf{T}\left(\Theta+\vartheta_{j}(t), \mathbf{R}+\mathbf{k}_{j}(t), I+\mathcal{S}+D \mathcal{V}\right)-\mathbf{T}_{g}(\Theta, \mathbf{R}, \mathbf{I})\right\|_{W^{1, p}(\Omega)} \leq \\
c\left\|\vartheta_{j}(t)\right\|_{W^{1, p}(\Omega)}+c\left\|\mathbf{k}_{j}(t)\right\|_{W^{1, p}(\Omega)}+c\left\|D \mathcal{V}\left(\vartheta_{j}, \mathbf{k}_{j}, t\right)\right\|_{W^{1, p}(\Omega)}+ \\
\left|\mathcal{S}\left(\vartheta_{j}, \mathbf{k}_{j}, t\right)\right| \leq c \varepsilon+c \rho
\end{array}
$$

Substituting (6.18) and (6.19) into (6.13), we arrive at the inequality

$$
\begin{array}{r}
\left\|\mathcal{M}\left(\vartheta_{1}, \mathbf{k}_{1}\right)-\mathcal{M}\left(\vartheta_{2}, \mathbf{k}_{2}\right)\right\|_{W^{1, p}(\Omega)} \leq c\left(\left\|\vartheta_{1}-\vartheta_{2}\right\|_{\mathbb{E}_{p}}+\right. \\
\left.\left\|\mathbf{k}_{1}-\mathbf{k}_{2}\right\|_{\mathbb{F}_{p}}\right)\left(\left|D \partial_{t} \mathbf{u}_{1}\right|+\left|D \partial_{t} \mathbf{u}_{2}\right|\right)+c(\varepsilon+\rho)\left|D \partial_{t} \mathbf{u}_{1}-D \partial_{t} \mathbf{u}_{2}\right|,
\end{array}
$$

which holds for a.e. $t \in(0, T)$. Recall that $\partial_{t} \mathbf{u}_{1}$ has the representation (6.14), where the operators $\mathcal{P}$ and $\mathcal{Q}_{i}$ satisfy inequalities (5.15)-(5.17). In particular, we have for a.e. $t \in[0, T]$,

$$
\begin{array}{r}
\left\|D \partial_{t} \mathbf{u}_{i}(t)\right\|_{W^{1, p}(\Omega)} \leq c \| \mathcal{P}\left(\vartheta_{i}, \mathbf{k}_{i}, t\right)+\mathcal{Q}_{1}\left(\vartheta_{i}, \mathbf{k}_{i}\right)\left[\partial_{t} \vartheta_{i}\right]+ \\
\mathcal{Q}_{2}\left(\vartheta_{i}, \mathbf{k}_{i}, t\right)\left[\partial_{t} \mathbf{k}_{i}(t)\right] \|_{W^{2, p}(\Omega)} \leq \\
c\left\|\partial_{t} \theta_{i}(t)\right\|_{W^{1, p}(\Omega)}+\left\|\partial_{t} \mathbf{k}_{i}(t)\right\|_{W^{1, p}(\Omega)}+c(\varrho+\varepsilon)
\end{array}
$$


Thus we get

$$
\begin{array}{r}
\left\|D \partial_{t} \mathbf{u}_{i}\right\|_{L^{p}\left(0, T ; W^{1, p}(\Omega)\right)} \leq c\left\|\partial_{t} \theta_{i}\right\|_{L^{p}\left(0, T ; W^{1, p}(\Omega)\right)}+c\left\|\partial_{t} \mathbf{k}_{i}\right\|_{L^{p}\left(0, T ; W^{1, p}(\Omega)\right)}+ \\
c(\varrho+\varepsilon) \leq c\left(\left\|\theta_{i}\right\|_{\mathbb{E}_{p}}+c\left\|\mathbf{k}_{i}\right\|_{\mathbb{F}_{p}}\right)+c(\varrho+\varepsilon) \leq c(\rho+\varepsilon) .
\end{array}
$$

The difference $\partial_{t} \mathbf{u}_{1}(t)-\partial_{t} \mathbf{u}_{2}(t)$ admits the representation

$$
\partial_{t} \mathbf{u}_{1}-\partial_{t} \mathbf{u}_{2}=I_{1}+I_{2}
$$

where

$$
\begin{gathered}
I_{1}=\mathcal{Q}_{1}\left(\vartheta_{1}, \mathbf{k}_{1}\right)\left[\partial_{t} \vartheta_{1}-\partial_{t} \vartheta_{2}\right]+\mathcal{Q}_{2}\left(\vartheta_{1}, \mathbf{k}_{1}, t\right)\left[\partial_{t} \mathbf{k}_{1}(t)-\partial_{t} \mathbf{k}_{2}\right], \\
I_{2}=\mathcal{P}\left(\vartheta_{1}, \mathbf{k}_{1}\right)-\mathcal{P}\left(\vartheta_{2}, \mathbf{k}_{2}\right)+\left(\mathcal{Q}_{1}\left(\vartheta_{1}, \mathbf{k}_{1}\right)-\mathcal{Q}_{1}\left(\vartheta_{2}, \mathbf{k}_{2}\right)\right)\left[\partial_{t} \vartheta_{2}\right]+ \\
\left(\mathcal{Q}_{2}\left(\vartheta_{1}, \mathbf{k}_{1}\right)-\mathcal{Q}_{2}\left(\vartheta_{2}, \mathbf{k}_{2}\right)\right)\left[\partial_{t} \mathbf{k}_{2}\right] .
\end{gathered}
$$

Arguing as in the proof of $(6.21)$, we obtain for a.e. $t \in(0, T)$,

$$
\begin{array}{r}
\left\|D I_{1}\right\|_{L^{p}\left(0, T ; W^{1, p}(\Omega)\right)} \leq c\left\|\partial_{t} \theta_{1}-\partial_{t} \theta_{2}\right\|_{L^{p}\left(0, T ; W^{1, p}(\Omega)\right)}+ \\
c\left\|\partial_{t} \mathbf{k}_{1}-\partial_{t} \mathbf{k}_{2}\right\|_{L^{p}\left(0, T ; W^{1, p}(\Omega)\right)} \leq c\left(\left\|\theta_{1}-\theta_{2}\right\|_{\mathbb{E}_{p}}+\left\|\mathbf{k}_{1}-\mathbf{k}_{2}\right\|_{\mathbb{F}_{p}}\right)
\end{array}
$$

Next, estimates (5.15)-(5.16) imply

$$
\begin{gathered}
\left\|I_{2}(t)\right\|_{W^{2, p}(\Omega)} \leq c\left(\left\|\vartheta_{1}(t)-\vartheta_{2}(t)\right\|_{W^{1, p}(\Omega)}+\right. \\
\left.\left\|\mathbf{k}_{1}(t)-\mathbf{k}_{2}(t)\right\|_{W^{1, p}(\Omega)}\right)\left(1+\left\|\partial_{t} \vartheta_{2}(t)\right\|_{W^{1, p}(\Omega)}+\left\|\partial_{t} \mathbf{k}_{2}(t)\right\|_{W^{1, p}(\Omega)}\right) \leq \\
c\left(\left\|\vartheta_{1}-\vartheta_{2}\right\|_{\mathbb{E}_{p}}+\left\|\mathbf{k}_{1}-\mathbf{k}_{2}\right\|_{\mathbb{F}_{p}}\right)\left(1+\left\|\partial_{t} \vartheta_{2}(t)\right\|_{L^{p}(\Omega)}+\left\|\partial \mathbf{k}_{2}(t)\right\|_{L^{p}(\Omega)}\right) .
\end{gathered}
$$

which gives

$$
\begin{array}{r}
\left\|D I_{2}(t)\right\|_{L^{p}\left(0, T ; W^{1, p}(\Omega)\right)} \leq c\left(\left\|\vartheta_{1}-\vartheta_{2}\right\|_{\mathbb{E}_{p}}+\left\|\mathbf{k}_{1}-\mathbf{k}_{2}\right\|_{\mathbb{F}_{p}}\right)(1+ \\
\left.\left\|\vartheta_{2}\right\|_{\mathbb{E}_{p}}+\left\|\mathbf{k}_{2}\right\|_{\mathbb{F}_{p}}\right) \leq c\left(\left\|\vartheta_{1}-\vartheta_{2}\right\|_{\mathbb{E}_{p}}+\left\|\mathbf{k}_{1}-\mathbf{k}_{2}\right\|_{\mathbb{F}_{p}}\right)
\end{array}
$$

Substituting (6.25) and (6.24) into (6.23), we obtain

$$
\left\|D \partial_{t} \mathbf{u}_{1}-D \partial_{t} \mathbf{u}_{2}\right\|_{L^{p}(Q)} \leq c\left(\left\|\vartheta_{1}-\vartheta_{2}\right\|_{\mathbb{E}_{p}}+\left\|\mathbf{k}_{1}-\mathbf{k}_{2}\right\|_{\mathbb{F}_{p}}\right)
$$

Combining this result with (6.22) and (6.20), we obtain the desired estimate (6.9). Finally, notice that

$\left\|\mathcal{M}\left(\vartheta_{i}, \mathbf{k}_{i}\right)\right\|_{L^{p}\left(0, T ; W^{1, p}(\Omega)\right)} \leq\left\|\mathbf{T}\left(\theta_{i}, \mathbf{K}_{i}, D \mathbf{u}_{i}\right)\right\|_{L^{p}\left(0, T ; W^{1, p}(\Omega)\right)}\left\|D \partial_{t} \mathbf{u}_{i}\right\|_{L^{p}\left(0, T ; W^{1, p}(\Omega)\right)}$.

Combining this result with (6.19) and (6.22), we obtain (6.10).

The next lemma concerns the maximal regularity results for parabolic boundary value problems 
Lemma 12 Let $\Omega$ be a bounded domain in $\mathbb{R}^{3}$ with $C^{\infty}$ boundary $\partial \Omega$ and $\Theta \in C^{\infty}(\Omega)$ be a strictly positive function. Then for every $T>0$ and $f \in$ $L^{p}(Q), Q=\Omega \times[0, T], p \in(3, \infty)$, the problem

$$
\begin{array}{r}
c_{T} \partial_{t} \vartheta-\Delta\left(\frac{\vartheta}{\Theta^{2}}\right)=f \text { in } Q, \\
\partial_{n} \vartheta=0 \quad \text { on } \partial \Omega \times(0, T), \quad \vartheta(x, 0)=0 \text { in } \Omega,
\end{array}
$$

has a unique solution satisfying the inequality

$$
\|\vartheta\|_{\mathbb{E}_{p}} \leq c\|f\|_{L^{p}\left(0, T ; W^{1, p}(\Omega)\right)},
$$

where $c$ depends only on $\Omega, T, p$ and $\Theta$.

Proof The existence of solution $\vartheta \in L^{p}\left(0, T ; W^{3, p}(\Omega)\right)$ with $\partial u_{t} \in L^{p}\left(0, T ; W^{1, p}(\Omega)\right)$ follows from the general theory of parabolic equations see [22], Theorem 5.4. Hence it suffices to prove estimate (6.27). Since $\partial \Omega$ belongs to the class $C^{\infty}$, we can introduce the normal coordinates in a neighborhood $\partial \Omega$, see [17],ch.13. It follows that there is a collection of linearly independent differential operators $\mathbf{a}_{i}(x) \nabla, i=1,2,3$ such that $\mathbf{a}_{i} \in C_{0}^{\infty}(\Omega)$, and

$$
\mathbf{a}_{1}=\mathbf{n}, \quad \mathbf{a}_{i} \cdot \mathbf{n}=0, \quad i=2,3 \text { on } \partial \Omega .
$$

For every integer $l \geq 1$ and for all $\vartheta \in W^{l, p}(\Omega)$, we have

$$
\|\varrho\|_{W^{l, p}(\Omega)} \leq c\left(l, \mathbf{a}_{i}\right) \sum_{i}\left\|\mathbf{a}_{i} \cdot \nabla \varrho\right\|_{W^{l-1, p}(\Omega)}+c(l)\|\varrho\|_{W^{l-1, p}(\Omega)}
$$

It follows from the maximal regularity results for parabolic boundary value problems, see [4] and [2], that for every $f \in L^{p}(Q)$ problem (6.27) has a unique solution satisfying the inequality

$$
\|\vartheta\|_{L^{p}\left(0, T ; W^{2, p}(\Omega)\right)}+\left\|\partial_{t} \vartheta\right\|_{L^{p}(Q)}+\|\vartheta\|_{C\left(0, T ; D_{p}(\Delta)\right)} \leq c\|f\|_{L^{p}(Q)} .
$$

Here $D_{p}(\Delta)$ is the subspace of the Besov space $B_{p, p}^{2-1 / p}(\Omega)$ which consists of all functions $\vartheta \in B_{p, p}^{2-1 / p}(\Omega)$ such that $\partial_{n} \vartheta=0$ on $\Omega$. Since the embedding $B_{p, p}^{2-1 / p}(\Omega) \hookrightarrow B_{p, p}^{1}(\Omega)=W^{1, p}(\Omega)$ is bounded, inequality (6.29) implies the estimate

$$
\|\vartheta\|_{L^{p}\left(0, T ; W^{2, p}(\Omega)\right)}+\left\|\partial_{t} \vartheta\right\|_{L^{p}(Q)}+\|\vartheta\|_{L^{\infty}\left(0, T ; W^{1, p}(\Omega)\right.} \leq c\|f\|_{L^{p}(Q)}
$$

The same conclusion can be drawn if if we replace the Neumann boundary condition in (6.26) by the Dirichlet boundary condition $\vartheta=0$ on $\partial \Omega \times(0, T)$. Now introduce the functions $\vartheta_{i}=\mathbf{a}_{i} \nabla \vartheta$. It follows from (6.28) that

$$
\begin{aligned}
\|\vartheta\|_{\mathbb{E}_{p}} \leq & c \sum_{i}\left(\left\|\vartheta_{i}\right\|_{L^{p}\left(0, T ; W^{2, p}(\Omega)\right)}+\left\|\partial_{t} \vartheta_{i}\right\|_{L^{p}(Q)}+\left\|\vartheta_{i}\right\|_{L^{\infty}\left(0, T ; W^{1, p}(\Omega)\right.}\right)+ \\
& c\left(\|\vartheta\|_{L^{p}\left(0, T ; W^{2, p}(\Omega)\right)}+\|\vartheta\|_{L^{p}(Q)}+\|\vartheta\|_{L^{\infty}\left(0, T ; W^{1, p}(\Omega)\right.}\right)
\end{aligned}
$$


Next, applying the operators $\mathbf{a}_{i} \cdot \nabla$ to both sides of (6.26) we obtain the equations

$$
\begin{array}{r}
c_{T} \partial_{t} \vartheta_{i}-\Delta\left(\frac{\vartheta_{i}}{\Theta^{2}}\right)=f_{i} \text { in } Q, \\
\vartheta_{1}=0, \quad \partial_{n} \vartheta_{i}=0, i=2,3 \text { on } \partial \Omega \times(0, T), \\
\vartheta_{i}(x, 0)=0, \quad i=1,2,3 \text { in } \Omega,
\end{array}
$$

where

$$
f_{i}=\mathbf{a}_{i} \nabla f+\mathbf{a}_{i} \Delta\left(\vartheta \nabla\left(\frac{1}{\Theta^{2}}\right)\right)-\partial_{k}\left(\frac{1}{\Theta^{2}} \partial_{k} \mathbf{a}_{i} \cdot \nabla \vartheta\right)-\partial_{k} \mathbf{a}_{i} \cdot \partial_{k}\left(\frac{1}{\Theta^{2}} \nabla \vartheta\right)
$$

Since $\mathbf{a}_{i}$ and $\Theta^{-2}$ belong to the class $C^{\infty}(\Omega)$, we have

$$
\left\|f_{i}\right\|_{L^{p}(\Omega \times(0, T))} \leq c\|f\|_{L^{p}\left(0, T ; W^{1, p}(\Omega)\right)}+c\|\vartheta\|_{L^{p}\left(0, T ; W^{2, p}(\Omega)\right)}
$$

From this and maximal regularity estimate (6.30) with $\vartheta$ and $f$ replaced by $\vartheta_{i}$ and $f_{i}$ we obtain

$$
\begin{array}{r}
\left\|\vartheta_{i}\right\|_{L^{p}\left(0, T ; W^{2, p}(\Omega)\right)}+\left\|\partial_{t} \vartheta_{i}\right\|_{L^{p}(Q)}+\left\|\vartheta_{i}\right\|_{L^{\infty}\left(0, T ; W^{1, p}(\Omega)\right.} \leq \\
c\|f\|_{L^{p}\left(0, T ; W^{1, p}(\Omega)\right)}+c\|\vartheta\|_{L^{p}\left(0, T ; W^{2, p}(\Omega)\right)}
\end{array}
$$

which along with (6.29) implies

$$
\left\|\vartheta_{i}\right\|_{L^{p}\left(0, T ; W^{2, p}(\Omega)\right)}+\left\|\partial_{t} \vartheta_{i}\right\|_{L^{p}(Q)}+\left\|\vartheta_{i}\right\|_{L^{\infty}\left(0, T ; W^{1, p}(\Omega)\right.} \leq c\|f\|_{L^{p}\left(0, T ; W^{1, p}(\Omega)\right)}
$$

Substituting this inequality and inequality (6.29) into (6.31) we obtain desired estimate (6.27).

We are now in a position to complete the proof of Theorem 1. Fix $\rho_{0}$ and $\varepsilon_{0}$ meeting all requirements of Lemmas 9-11. Next, consider the mapping $\mathfrak{F}:(\tilde{\vartheta}, \tilde{\mathbf{k}}) \mapsto(\vartheta, \mathbf{k})$ defined as a solution to the boundary value problem

$$
\begin{array}{r}
c_{T} \frac{\partial \vartheta}{\partial t}-\kappa \Delta\left(\frac{\vartheta}{\Theta^{2}}\right)=\mathcal{N}_{0}+\sum_{1}^{2} \mathcal{N}_{i}(\tilde{\vartheta}, \mathbf{k})+\mathcal{M}(\tilde{\vartheta}, \mathbf{k}) \text { in } Q, \\
\partial_{n} \vartheta=0 \text { on } \partial \Omega \times(0, T), \quad \vartheta(x, 0)=0 \text { in } \Omega, \\
\mathbf{k}(x, t)=k_{0}(x)+\int_{0}^{t} \chi(s) \mathcal{G}(\tilde{\vartheta}(s), \tilde{\mathbf{k}}(s)) d s \text { in } Q .
\end{array}
$$

In view of Lemmas 9-11, the mapping $\mathfrak{F}: \Gamma(\rho) \rightarrow \mathbb{E}_{p} \times \mathbb{F}_{p}$ is well defined for all $\rho \in\left(0, \rho_{0}\right]$ and $\varepsilon \in\left(0, \varepsilon_{0}\right]$. It follows from inequality (6.12) in Lemma 11 that for every $(\tilde{\vartheta}, \tilde{\mathbf{k}}) \in \Gamma(\rho)$, the function $\mathbf{k}$ given by $(6.33 \mathrm{c})$ satisfies the inequalities

$$
\begin{array}{r}
\|\mathbf{k}\|_{L^{\infty}\left(0, T ; W^{1, p}(\Omega)\right)} \leq\left\|\mathbf{k}_{0}\right\|_{W^{1, p}(\Omega)}+c T_{0}, \\
\left\|\partial_{t} \mathbf{k}\right\|_{L^{p}\left(0, T ; W^{1, p}(\Omega)\right)} \leq c T_{0}^{1 / p}
\end{array}
$$


since $\chi(t)$ vanishes for $t>T_{0}$. Recalling Definition 7 of the space $\mathbb{F}_{p}$, we obtain

$$
\|\mathbf{k}\|_{\mathbb{F}_{p}} \leq c\left(T_{0}+T_{0}^{1 / p}+\left\|\mathbf{k}_{0}\right\|_{W^{1, p}(\Omega)}\right) .
$$

Choose $T_{0}$ and $\mathbf{k}_{0}$ so small that $c\left(T_{0}+T_{0}^{1 / p}+\left\|\mathbf{k}_{0}\right\|_{W^{1, p}(\Omega)}\right) \leq \rho$. Thus we get

$$
\|\mathbf{k}\|_{\mathbb{F}_{p}} \leq \rho .
$$

Repeating these arguments and applying inequality (6.11) in Lemma 11, we conclude that for $\left(\tilde{\vartheta}_{i}, \tilde{\mathbf{k}}_{i}\right) \in \Gamma(\rho), i=1,2$, the corresponding functions $\mathbf{k}_{i}$ satisfy the inequality

$$
\left\|\mathbf{k}_{1}-\mathbf{k}_{2}\right\|_{\mathbb{F}_{p}} \leq c\left(T_{0}+T_{0}^{1 / p}\right)\left(\left\|\tilde{\mathbf{k}}_{1}-\tilde{\mathbf{k}}_{2}\right\|_{\mathbb{F}_{p}}+\left\|\tilde{\vartheta}_{1}-\tilde{\vartheta}_{2}\right\|_{\mathbb{E}_{p}}\right) .
$$

Notice that for such a choice $T_{0}$ and $\mathbf{k}_{0}$, the couple $(\tilde{\vartheta}, \mathbf{k}) \in \Gamma(\rho)$. Hence we can estimate the right hand side of (6.33a) using Lemmas 10 and 11. It follows from inequalities (6.7), (6.8) in Lemma 10, inequality (6.10) in Lemma 11 and inequality (6.27) in Lemma 6.6 that for every $(\tilde{\vartheta}, \tilde{\mathbf{k}}) \in \Gamma(\rho)$ and $\mathbf{k}=\mathbf{k}(\tilde{\vartheta}, \tilde{\mathbf{k}})$, a solution to problem (6.33a)-(6.33b) satisfies the inequality

$$
\|\vartheta\|_{\mathbb{E}_{p}} \leq c \rho(\rho+\varepsilon)
$$

Hence the mapping $\mathfrak{F}$ takes the ball $\Gamma(\rho)$ into itself for $c(\rho+\varepsilon) \leq 1$. Next, inequality (6.6) in Lemma 10, inequality (6.9) in Lema 11 and inequality (6.27) in Lemma 6.6 imply that for every $\left(\tilde{\vartheta}_{i}, \tilde{\mathbf{k}}_{i}\right) \in \Gamma(\rho)$ and $\mathbf{k}_{i}=\mathbf{k}\left(\tilde{\vartheta}_{i}, \tilde{\mathbf{k}}_{i}\right)$ the corresponding solutions $\vartheta_{i}$ to to problem (6.33a)-(6.33b) satisfy

$$
\left\|\vartheta_{1}-\vartheta_{2}\right\|_{\mathbb{E}_{p}} \leq c(\rho+\varepsilon)\left(\left\|\tilde{\vartheta}_{1}-\tilde{\vartheta}_{2}\right\|_{\mathbb{E}_{p}}+\left\|\mathbf{k}_{1}-\mathbf{k}_{2}\right\|_{\mathbb{F}_{p}}\right) .
$$

Combining this result with (6.36) and noting that $c\left(T_{0}+T_{0}^{1 / p}\right) \leq \rho$, we finally arrive at the inequality

$$
\left\|\vartheta_{1}-\vartheta_{2}\right\|_{\mathbb{E}_{p}}+\left\|\mathbf{k}_{1}-\mathbf{k}_{2}\right\|_{\mathbb{F}_{p}} \leq c(\rho+\varepsilon)\left(\left\|\tilde{\vartheta}_{1}-\tilde{\vartheta}_{2}\right\|_{\mathbb{E}_{p}}+\left\|\tilde{\mathbf{k}}_{1}-\tilde{\mathbf{k}}_{2}\right\|_{\mathbb{F}_{p}}\right) .
$$

Hence, for $c(\rho+\varepsilon) \leq 1$ the mapping $\mathfrak{F}: \Gamma(\rho) \rightarrow \Gamma(\rho)$ is a contraction. Therefore, it has a unique fixed point $(\vartheta, \mathbf{k})$ in this ball. Obviously $(\vartheta, \mathbf{k})$ satisfies equation (6.33). Applying Theorem 3, we conclude that that functions $(\vartheta, \mathbf{k}), \mathbf{v}=\mathcal{V}(\vartheta, \mathbf{k}$ and the matrix $\mathbf{S}=\mathcal{S}(\vartheta, \mathbf{k})$ serve as a solution to the modified problem (4.5). On the other hand, in view of Theorem 3 , the corrector $\mathcal{E}$ in the momentum balance equation (4.5c) equals zero. Hence the functions $(\vartheta, \mathbf{k})$ and $\mathbf{u}=\operatorname{Id}+\mathbf{S} x+\mathbf{v}$ form a solution to the main problem (3.5)-(3.6). This completes the proof of Theorem 1 . 


\section{Conluding remarks}

The obtained results show that the nonlinear growth models proposed in mechanics and biology are well posed from the mathematical point of view. The models admit the local in time, classical solutions. To our best knowledge there are no such results in the mathematical literature of the subject due to the complexity of the nonlinear, coupled models. The subsequent papers would be devoted to the further analysis of the models and some applications as well as to the numerical solutions of the models. This field of research is important for real life problems in mechanics, biology and medicine.

\section{References}

1. Alford P, Humphrey J, Taber L. Growth and remodeling in a thick-walled artery model: effects of spatial variations in wall constituents. Biomech. and model. in mecha., 7 (4): 245-262.

2. Amman H., Linear and quasilinear parabolic problems, I. Bisrkhauser Verlag, Basel,1995

3. Ciarlet P., Mathematical elasticity, volume 1: Three-dimensional Elasticity, xiii+451, Elsevier Science Publishers B.V. (1988)

4. Denk R., Hieber M., Prüss J., Optimal $L^{p}-L^{q}$-estimates for parabolic boundary value problems with inhomogeneous data. Math. Z. , 257, 193-224 (2007)

5. De Cristoforis M. Lanza, Valent T., On Neumann's problem for a quasilinear differential system of the finite elastostatics type. Local theorems of existence and uniqueness. Rend. Sem. Mat. Univ. Padova, 68 , 183-206 (1982)

6. De Pascalis R., Destrade M., and Goriely A., Nonlinear Correction to the Euler Buckling Formula for Compressed Cylinders with Guided-Guided End Conditions. Journal of Elasticity, (2011) 102(2): 191-200.

7. Epstein M, Maugin, G. Thermomechanics of volumetric growth in uniform bodies, Int. J. Plasticity 16: 951-978.

8. Eshelby, J. D., 1951. The force on an elastic singularity. Phil. Trans. R. Soc. A 244: 87-112.

9. J.F. Ganghoffer. On Eshelby tensors in the context of open systems: application to volumetric growth. Int. J. Engng Sci. doi: 10.1016/j.ijengsci.2010.04.003.

10. J.F. Ganghoffer, 2011. Mechanical modeling of growth considering domain variation Part II: volumetric and surface growth involving Eshelby tensors. J. Mech. Phys. Solids, doi: $10.1016 / \mathrm{j} / \mathrm{jmps} / 2010.05 .003$.

11. Gurtin M., Spector S., On stability and uniqueness in finite elasticity. Arch. Rational Mech. Anal., 70, 153-165 (1979)

12. Kuhl, E., Maas, R., Himpel, G., Menzel, A., 2007. Computational modeling of arterial wall growth. Biomech. and Model. in Mechanobiol., 6 (5): 321-331.

13. Menzel, A., 2007. A fibre reorientation model for orthotropic multiplicative growth. Biomech. and Modeling in Mechanobiol. 6 (5): 303-320.

14. Menzel, A., Kuhl, E., 2012. Frontiers in growth and remodeling. Mech. Res. Comm. 42: $1-14$.

15. D. Moulton, D., Goriely, A., 2011 Anticavitation and differential growth in elastic shellsJ. Elasticity, 102(2), 117-132.

16. Olsson, T., Klarbring, A., 2008. Residual stresses in soft tissue as a consequence of growth and remodeling : application to an arterial geometry. Eur. J. Mech./A Solids 27 (6): 959-974.

17. Plotnikov P., Sokołowski J., (2012) Compressible Navier-Stokes equations. Theory a nd shape optimization. Mathematical Institute of the Polish Academy of Sciences. Mathematical Monographs (New Series)], 73. Birkhäuser/Springer Basel AG, Basel, 2012. xvi+457 pp. ISBN: 978-3-0348-0366-3. 
18. Pompe W., 2003, Korn's First Inequality with variable coefficients and its generalization, Comment.Math.Univ.Carolin. 44, 57-70.

19. Rodriguez E.K., Hoger A., McCulloch A.D., Stress-dependant finite growth in soft elastic tissues, J. Biomech. 27: 455-467.

20. Rodriguez, E., Hoger, A., McCulloch, A., 1994. Stress-dependent finite growth law in soft elastic tissue. J. Biomech. 27: 455-467.

21. Rodriguez, J., Goicolea, J., Gabaldón, F., 2007. A volumetric model for growth of arterial walls with arbitrary geometry and loads. J. Biomech. 40 (5): 961-971.

22. Solonnikov V.A., On boundary value problems for linear parabolic systems of differential equations of general form. Trudy Mat. Inst. Steklov, LXXXIII: 1-159 (1965)

23. Stopelli F., Un teorema di esistenza ed unicita relativo alle equazioni dell'elastostatica isoterma per deformazioni finite, Ricerche Matematiche, 3, 247-267 (1954)

24. Taber, L., 1995. Biomechanics of growth, remodeling and morphogenesis. Appl. Mech. Rev. 48: 48754.

25. Taber, L., 1998. A model for aortic growth based on fluid shear and fiber stresses. J. Biomech. Engng, 120: 348-354.

26. Thompson J., Some existence theorems for the traction boundary value problem of linearized elastostatics, Arch. Rational Mech. Anal., 32, 369-399 (1969)

27. Valent T., Boundary value problems of finite elasticity. Local theorems on existence, uniqueness, and analytic dependence on data Springer Tracts in Natural Philosophy, , xii+191, Springer-Verlag, New York (1988)

28. Vignes, C., Papadopoulos, P., 2010. Material growth in thermoelastic continua : Theory, algorithmics, and simulation. Computer Methods in Applied Mechanics and Engng 199: 979-996. 